\title{
A SUBSTRUCTURAL LOGIC FOR LAYERED GRAPHS
}

\author{
MATTHEW COLLINSON, KEVIN MCDONALD, AND DAVID PYM
}

\begin{abstract}
Complex systems, be they natural or synthetic, are ubiquitous. In particular, complex networks of devices and services underpin most of society's operations. By their very nature, such systems are difficult to conceptualize and reason about effectively. The concept of layering is widespread in complex systems, but has not been considered conceptually. Noting that graphs are a key formalism in the description of complex systems, we establish a notion of a layered graph. We provide a logical characterization of this notion of layering using a non-associative, non-commutative substructural, separating logic. We provide soundness and completeness results for a class of algebraic models that includes layered graphs, which give a mathematically substantial semantics to this very weak logic. We explain, via examples, applications in information processing and security.
\end{abstract}

\section{INTRODUCTION}

Complex systems, be they natural or synthetic, are ubiquitous. In particular, complex networks of devices and services underpin most of society's operations. By their very nature, such systems are difficult to conceptualize and reason about effectively.

One seemingly natural notion, which helps to manage complexity and which is commonly found in discussions of complex systems, is that of layering: the system is considered to consist of a collection of interconnected layers that have distinct, identifiable roles in the overall operations of the system.

Examples of systems that are naturally layered include the IP stack, many computer and information security architectures, and transport infrastructure systems. Layers can be informational or physical, and a given system may combine both kinds. For example, a transport system might be considered to have an infrastructure layer (e.g., roads, railway lines), a timetable layer (e.g., buses and trains arriving/departing given locations in the infrastructure), and a social layer (e.g., the groupings and movements of people enabled by the transport services).

To understand and reason about complex systems, we typically seek to construct mathematical models. A number of approaches are possible, and the literature is vast. Many techniques draw upon mathematical analysis and the theory of (possibly stochastic) differential and integral equations. Other techniques employ discrete event simulation tools. In neither of these literatures has the concept of layering been drawn out and studied explicitly, although it is widely used implicitly. In both approaches, the primary focus tends to be on the dynamics of the systems being studied.

To understand the concept of layering, it is necessary to have an approach to modelling that makes explicit the structural, as well as the dynamic, aspects of systems. In recent work, some of us have developed a compositional approach to mathematical systems modelling that is based on concepts of process (capturing the services delivered by the system as well as its overall evolution), resource, location, and environment and which uses mathematical techniques from process algebra, logic, and probability theory $[13,11,14,12]$. Associated with this work is a tool, Core Gnosis $[14,12]$, which implements these concepts rather closely, and which has been employed in a wide range of industry-based studies [12], most of which are security-related.

Underpinning this work are two semantic judgements,

$$
L, R, E \stackrel{a}{\longrightarrow} L^{\prime}, R^{\prime}, E^{\prime} \quad \text { and } \quad L, R, E \vDash \phi,
$$

where $L$ denotes location, $R$ denotes resource, $E$ denotes a process, $a$ denotes an action (part of a process), and $\phi$ is a logical formula (in a modal substructural logic $[13,11,12]$ ). 
The first judgement is read as 'the process $E$ evolves by action $a$ with respect to resources $R$ at location $L$ to become the process $E^{\prime}$ executing with respect to resources $R^{\prime}$ at location $L^{\prime}$ '. The second is read, in the style of Hennessy-Milner logic [27, 12], as 'property $\phi$ holds of process $E$ executing with respect to resources $R$ at location $L$ '.

Here the concept of layering forms part of the model of location. In $[11,12]$, the required properties of a model of location are described axiomatically, but leading examples are given by various (directed) graph-theoretic constructions. There is very little work in the literature on layering in graphs. Notable exceptions are [36, 19, 37], which give direct definitions that are essentially examples of what we shall identify as a special kind of layered graph amounting to the intuitive notion of a 'stack'. Another exception is found [23, 24], in which graphs are replicated to form layered structures that can be understood as stacks. In [6, 29], multiple layers are considered to be given by multiple relations over a single set of nodes. Various more applied studies (e.g., [46] or many papers on network architectures) consider notions of layered models, typically without defining formally the concept of layering. It seems, however, that the key idea is to start with what it means for one graph (upper) to be layered over another (lower) graph, and for resources to flow from the upper graph to the lower one. Notions of layering appear to occur naturally in situations in which there are epistemic considerations on states of worlds; for example, with information sets in extensive game theory [5].

In this paper, we consider, in Section 2, what should be meant by a layered graph. In Section 3 , we use techniques from bunched logic $[35,41,20]$ to give a logical characterization of layering that uses 'separating connectives', in the sense of [44], to describe how properties of layers combine to give properties of the overall graph. This characterization makes use of a non-commutative, non-associative separating conjunction (and its associated implications). In Section 4, we set up a basic algebraic semantics for this logic, building on ideas from [17, 18]. We show that the class of models we employ includes the graphs considered in Section 2, and so establish a mathematically substantial semantics for this very weak logic.

Our logic is related to the system DW considered by Read [42], in which the multiplicative (intensional) conjunction (corresponding to the multiplicative bunching operation) is commutative and in which the multiplicative (intensional) implications - handed versions of linear implication, as present in Girard's Linear Logic [22], O'Hearn and Pym's BI [35], and Lambek's systems $[30,31,32,33]$ - that are naturally present in our system are absent. Read considers a hierarchy of extensions of DW, including axioms for associativity and many stronger structural properties. Read's hierarchy recovers the hierarchy of relevant systems [1, 2, 42, 43] up to Anderson and Belnap's R and classical logic itself. Just as in these systems, we work with a classical negation, deferring consideration of weaker systems of negation to another occasion.

Layering need not be defined in one direction only: it may be that two graphs are layered over each other. In modelling terms, this would mean that whilst it remains useful to separate the two layers, resources can flow both up and down. To this end, in Section 5, we establish a notion of 'bi-layering' that is consistent with our basic notion of layering. As an example, we recover the underlying structural concept of the definition of a layered graph given in [36] (we don't consider weighted edges, but do consider directedness, and do permit edges within layers). We also consider an important special case of layering, amounting to the intuitively understood notion of stacking. In Section 6, we consider logical characterizations of bi-layering and stacking.

To illustrate applicability, Section 7 considers three examples in detail which show how the logic can be used to detail practical problems. To facilitate giving the presentation of these examples, we enrich (informally) our logical language with some basic action modalities, with the evident intended semantics. More generally, this extension can be developed either in the style of dynamic logic [25] or in the Hennessy-Milner-style mentioned above [34, 12]. Both are beyond the scope of this paper and are deferred to another occasion. In computer science, motivated by the study of semi-structured data, Cardelli et al. [9] — in work that is similar to earlier work of Courcelle [15], with expressiveness and complexity studied by Dawar et al. [16] — have considered 'a spatial logic for querying graphs'. This logic is spatial in the sense that its multiplicative conjunction is interpreted as a composition of graphs, but this conjunction is both associative and commutative, and so not a candidate for a natural account of layering. Reynolds' Separation Logic [44], Ishtiaq 
and O'Hearn's Pointer Logic [28], and Gordon and Cardelli's Ambient Calculus [10] all employ constructs for spatial, graph-like models that are both associative and commutative. A comparison of all of these approaches with our work would be substantial further work and is beyond our present scope.

The first of our examples is the IP stack, where we use the logic to consider how an email message is sent from one user to another, as shown in Figure 1. When an email is sent, a user perceives the message as moving directly through the Client Layer. In practice the message moves into the IP layer, being split into packets and sent over the internet before being combined and displayed. Note that in Figure 1, Figure 3, and the corresponding and similar graphs in Section 7, we draw explicitly only the vertices, edges and associated actions that are relevant to the situation being described.

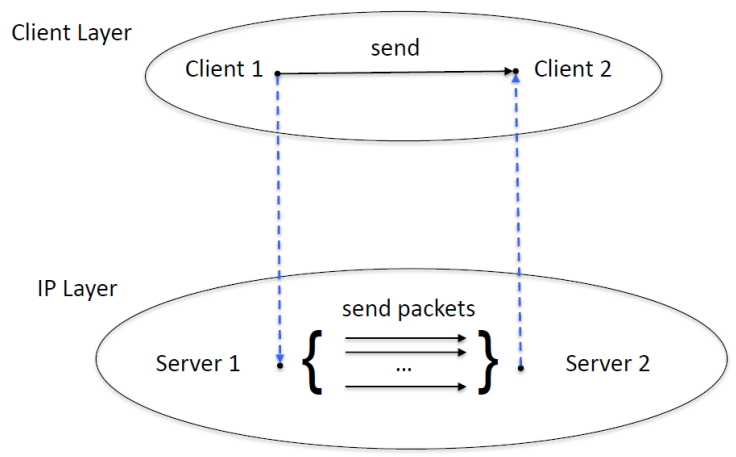

Figure 1. Email Clients and Servers in the IP Stack

Actions occur along edges as illustrated. In the Client Layer, for example, we have two vertices connected by an edge along which the send action can execute. In addition to send there are additional actions relating to the splitting, sending and reconstitution of the message. After logically describing properties that must hold as a result of these actions, we are able, using the layering conjunction, to show how the formulae combine to allow the sending of an email.

The second example is a security example about access control, using the layering conjunction to investigate avoiding a security barrier (see Bruce Schneier's website for a photograph). This scenario is shown in Figure 2 where an attempt at access control is being undermined by the missing part of the fence. We are able to capture the circumstances where a road user may pass through the barrier as intended, or avoid the security check and bypass the barrier by moving to another layer. In both cases the vehicle will be inside the barrier whether permitted or not. The use of the logic in this example means that we can construct a formula to succinctly represent the security flaw.

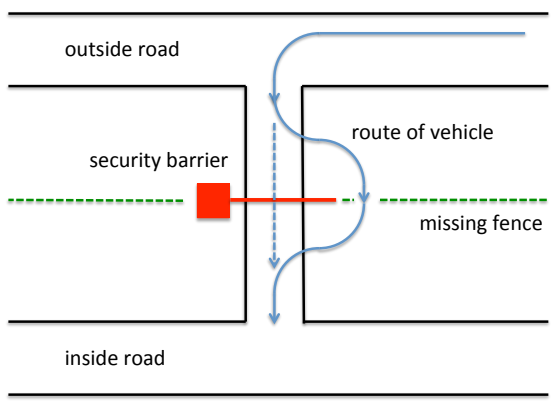

FiguRE 2. Security Barrier

The final example is also based on security, discussing flaws in the security policy of an organization. Consider an organization wishing to segregate data into secure and general partitions of 
their computer network, as shown in Figure 3. Policies can be written in an attempt to enforce this intention but they may be inadequate given the usage of mobile storage devices.

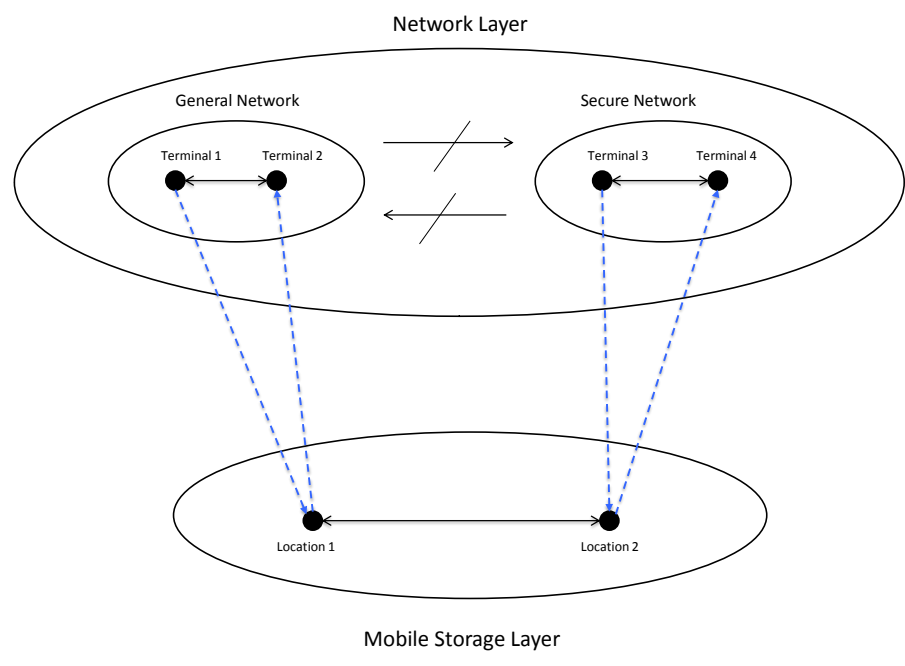

Figure 3. Data Segregation and Mobile Storage Graph

Mobile storage devices introduce an additional layer within the organizational structure and it is this additional layer that could lead to a breach of security policy. Secure data can be copied from the Secure Network, moved through the Mobile Layer and uploaded into the General Network. This example makes use of the implications associated with the conjunction meaning that we can determine situations that may lead to the security policy being violated.

In all examples, the non-commutativity and non-associativity of the conjunction plays a key role. Judgements stem from the way in which graphs combine to form layers and depend on properties specific to a given layer. Commutativity in particular would make it very difficult to consider any of the natural examples.

In Section 8 we establish soundness and completeness for the class of models introduced in Section 4 and conclude, in Section 9, with a section that establishes some basic proof theory for the logic. We give a sequent calculus and natural deduction system, showing their equivalence for provability. We also give a display calculus and use this to establish cut-elimination.

Note that many of the results contained in this paper have proofs that are simple consequences of the chosen definitions, or of a well-known form; such proofs have been omitted.

\section{A Layered Graph Construction}

Standard graph-theoretic notions are used throughout this paper. All graphs are assumed to be directed. For any graph $G$, let $V(G)$ be the set of vertices of $G, E(G)$ be the set of edges of $G$, and $\operatorname{Sg}(G)$ be the set of subgraphs of $G$. The notation $H \sqsubseteq G$ means $H \in \operatorname{Sg}(G)$.

We are often interested in situations in which we are given a graph $\mathcal{G}$ and a distinguished set of edges $\mathcal{E}$ of $\mathcal{G}$, and we then consider properties of the set of subgraphs of $\mathcal{G}$. In such a situation, we often refer to $\mathcal{G}$ as the ambient graph and $\mathcal{E}$ as the distinguished edge set. Let $G_{1}$ and $G_{2}$ be any two subgraphs of $\mathcal{G}$. The notation $G_{1} \rightsquigarrow \mathcal{E} G_{2}$ is used to signify the fact that $G_{2}$ is reachable from $G_{1}$ via an edge of $\mathcal{E}$. The notation $\not \varkappa_{\mathcal{E}}$ is the complement of the relation $\rightsquigarrow \mathcal{E}$. Let $G_{1}$ and $G_{2}$ be any two such subgraphs. Write $\mathcal{G}\left(G_{1}, G_{2}\right)$ for the set of edges of the ambient graph that connect any vertex of $G_{1}$ to any vertex of $G_{2}$. Define $\mathcal{G}\left[G_{1}, G_{2}\right]=\mathcal{G}\left(G_{1}, G_{2}\right) \cup \mathcal{G}\left(G_{2}, G_{1}\right)$.

We make use of partially-defined functions and expressions throughout. The notation $X \downarrow$ means that a given expression $X$ defines a value; the notation $X \uparrow$ means that it is undefined. Kleene equality will also be used: for any two (comparable) expressions $X$ and $Y$, we write $X \simeq Y$ to mean that either $X$ and $Y$ are both undefined, or that $X$ and $Y$ both define the same value. 

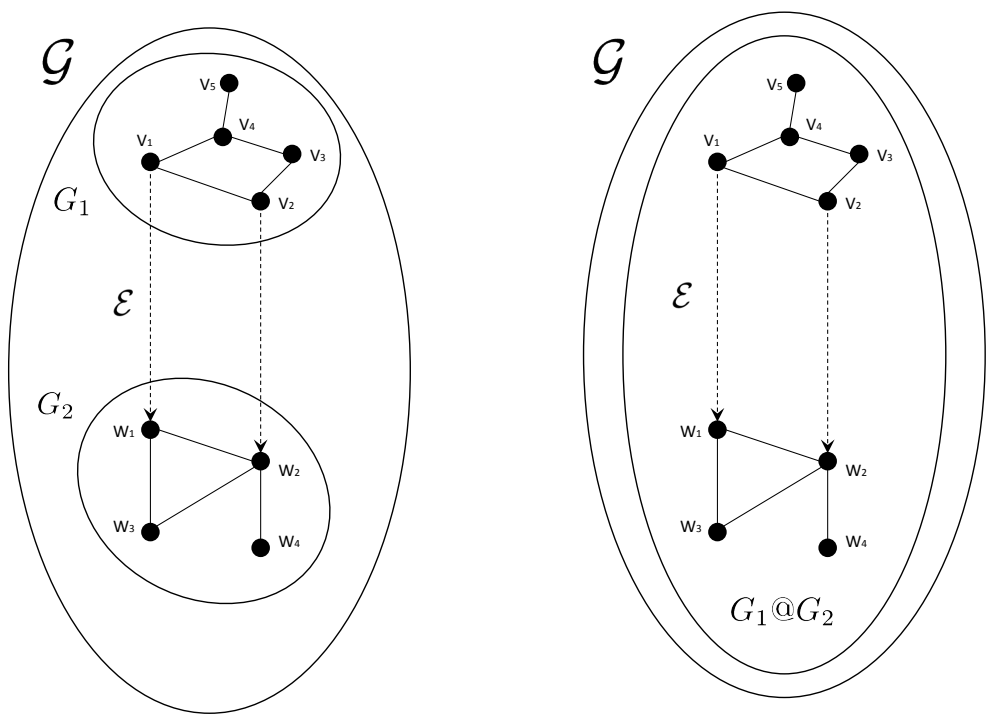

FiguRE 4. Layer Construction of Example 2.1

Given a graph $\mathcal{G}$ and a distinguished set of edges $\mathcal{E}$, we define a partial, binary operation $@: \mathcal{G} \times \mathcal{G} \longrightarrow \mathcal{G}$ as follows. For any subgraphs $G_{1}$ and $G_{2}$ of $\mathcal{G}$, the value $G_{1} @ G_{2}$ is defined if and only if $V\left(G_{1}\right) \cap V\left(G_{2}\right)=\emptyset$ and $G_{1} \rightsquigarrow \mathcal{E} G_{2}$ and $G_{2} \not{ }_{\mathcal{E}} G_{1}$. When defined, $G_{1} @ G_{2}$ is given by

$$
V\left(G_{1} @ G_{2}\right)=V\left(G_{1}\right) \cup V\left(G_{2}\right) \text { and } E\left(G_{1} @ G_{2}\right)=E\left(G_{1}\right) \cup E\left(G_{2}\right) \cup\left(\mathcal{E} \cap \mathcal{G}\left[G_{1}, G_{2}\right]\right) \text {. }
$$

When $G_{1} @ G_{2}$ is defined, it gives the (disjoint) union of the graph arguments, together with the edges of $\mathcal{E}$ between $G_{1}$ and $G_{2}$. The definition of this operator is relative to the given ambient graph $\mathcal{G}$ and distinguished set of edges $\mathcal{E}$. Where the distinguished set of edges $\mathcal{E}$ needs to be emphasized we write @E and we say that a graph $G$ is layered if $G=G_{1} @ G_{2}$, for some $G_{1}, G_{2}$.

Example 2.1. Let $1 \leq k \leq m, n$. Let $G_{1}$ and $G_{2}$ be graphs with $V\left(G_{1}\right)=\left\{v_{1}, \ldots, v_{m}\right\}, V\left(G_{2}\right)=$ $\left\{w_{1}, \ldots, w_{n}\right\}$ and $V\left(G_{1}\right) \cap V\left(G_{2}\right)=\emptyset$. Consider the set of pairs $S=\left\{\left(v_{i}, w_{i}\right) \mid 1 \leq i \leq k\right\}$. Define a set of distinguished, directed edges $\mathcal{E}$ to be given by the set of pairs $S$ : that is, the source of each edge takes the form $v_{i}$ and the target takes the form $w_{i}$ for some $1 \leq i \leq k$. Let $\mathcal{G}$ be the graph with $V(\mathcal{G})=V\left(G_{1}\right) \cup V\left(G_{2}\right)$ and $E(\mathcal{G})=E\left(G_{1}\right) \cup E\left(G_{2}\right) \cup \mathcal{E}$. Then $G_{1} @ G_{2}$ is defined (with respect to $\mathcal{G}$ and $\mathcal{E})$ and $G_{1} @ G_{2}=\mathcal{G}$. This construction is shown in Figure 4 for $m=5, n=4$.

We say that a pair $(\mathcal{G}, \mathcal{E})$ is a scaffold if it has at least one pair of subgraphs $G_{1}, G_{2}$ with $G_{1} @ G_{2}$ defined. Scaffolds are intended to highlight the ambient graph structure and edge set that may generate a particular layered graph. Different combinations of graphs and edges can produce different occurrences of layered graphs. The following result is immediate:

Proposition 2.2. Let $\mathcal{G}$ be a graph, and $\mathcal{E}$ a set of edges of $\mathcal{G}$. Then $(\mathcal{G}, \mathcal{E})$ is a scaffold if and only if there is a pair of vertices $v, w$ of $\mathcal{G}$ with an edge of $\mathcal{E}$ from $v$ to $w$, but no edge of $\mathcal{E}$ from $w$ to $v$.

Proposition 2.3. Let $(\mathcal{G}, \mathcal{E})$ be a scaffold, and $G_{1}, G_{2}$ be subgraphs of $\mathcal{G}$.

(1) There is neither a left-unit, nor a right-unit for the operation @.

(2) The value $G_{1} @ G_{1}$ is not defined, so the operation @ is not idempotent (up to Kleene equality, $\simeq$ ).

(3) The operation @ is not commutative (up to Kleene equality). Moreover, if $G_{1} @ G_{2}$ is defined, then $G_{2} @ G_{1}$ is not defined. We say that this makes @ contra-commutative.

(4) If $G_{1} \sqsubseteq G_{1}^{\prime}$ and $G_{2} \sqsubseteq G_{2}^{\prime}$, and $G_{1} @ G_{2}$ and $G_{1}^{\prime} @ G_{2}^{\prime}$ are both defined, then $G_{1} @ G_{2} \sqsubseteq$ $G_{1}^{\prime} @ G_{2}^{\prime}$.

Proof. (1) A left-unit $I$ would need to have $I @ O=O$, where $O$ is the empty graph, but $I @ O$ is undefined. Similarly, no right-unit can exist. 
(2) No subgraph can be disjoint from itself.

(3) If $G_{1} @ G_{2}$ is defined, then there is an edge of $\mathcal{E}$ from $G_{1}$ to $G_{2}$, so that $G_{2} @ G_{1}$ cannot be defined.

(4) Under the given conditions: $V\left(G_{1} @ G_{2}\right)=V\left(G_{1}\right) \cup V\left(G_{2}\right) \subseteq V\left(G_{1}^{\prime}\right) \cup V\left(G_{2}^{\prime}\right)=V\left(G_{1}^{\prime} @ G_{2}^{\prime}\right)$ and $E\left(G_{1} @ G_{2}\right)=E\left(G_{1}\right) \cup E\left(G_{2}\right) \cup\left(\mathcal{E} \cap \mathcal{G}\left[G_{1}, G_{2}\right]\right) \subseteq E\left(G_{1}^{\prime}\right) \cup E\left(G_{2}^{\prime}\right) \cup\left(\mathcal{E} \cap \mathcal{G}\left[G_{1}^{\prime}, G_{2}^{\prime}\right]\right)=$ $E\left(G_{1}^{\prime} @ G_{2}^{\prime}\right)$.

The notion of one graph being layered over another, and the applications that we pursue, suggest that the corresponding graph-theoretic definition should be directed. Thus, when we compose two graphs to form a layered graph, the composite should retain the directedness. We therefore seek a composition operation that is non-commutative. Proposition 2.3(3) shows that the scaffold definition (together with the definition of @) is sufficient to give such a composition, based on a simple idea of graph union. Note also that if a pair $(\mathcal{G}, \mathcal{E})$ is not a scaffold, then the operation @ is uninteresting since it is undefined on all arguments.

The operation @ is not necessarily associative, as the following example shows:

Example 2.4. Let $\mathcal{G}$ be the graph defined by $V(\mathcal{G})=\{t, u, v, w\}$ and $E(\mathcal{G})=\{(t, v),(u, w)\}$. Let $\mathcal{E}=E(\mathcal{G})$. Let $G_{1}, G_{2}, G_{3}$ be the subgraphs of $\mathcal{G}$ defined by $V\left(G_{1}\right)=\{t, u\}, V\left(G_{2}\right)=\{v\}$ and $V\left(G_{3}\right)=\{w\}$. Then $(\mathcal{G}, \mathcal{E})$ is a scaffold and $\left(G_{1} @ G_{2}\right) @ G_{3}$ is defined, but $G_{1} @\left(G_{2} @ G_{3}\right)$ is undefined.

Let $\mathcal{G}$ be a graph, and let $\mathcal{E}$ be a distinguished edge set. Let $\widetilde{\mathcal{G}}$ be a new graph, and let $\widetilde{\mathcal{E}}$ be a new distinguished set of edges as follows. Let $V(\widetilde{\mathcal{G}})=V(\mathcal{G})$. Let $\widetilde{\mathcal{E}}$ be the set of edges formed by taking each edge of $\mathcal{E}$ and swapping its source and target vertices, that is, by reversing its direction. Let $E(\widetilde{\mathcal{G}})=(E(\mathcal{G}) \backslash \mathcal{E}) \cup \widetilde{\mathcal{E}}$. Thus $\widetilde{\mathcal{G}}$ is formed by simply reversing all arrows of $\mathcal{E}$. For any subgraph $G$ of $\mathcal{G}$, let $\widetilde{G}$ be the subgraph of $\widetilde{\mathcal{G}}$ formed by reversing all edges of $\mathcal{E}$ that lie in $G$. Further to the contra-commutativity of the graph composition, @, we have:

Proposition 2.5. If the pair $(\mathcal{G}, \mathcal{E})$ is a scaffold, then $(\widetilde{\mathcal{G}}, \widetilde{\mathcal{E}})$ is also a scaffold. Let $G_{1}, G_{2}$ be subgraphs of $\mathcal{G}$. Then $G_{1} @ G_{2}$ is defined with respect to the scaffold $(\mathcal{G}, \mathcal{E})$ iff $\widetilde{G_{2}} @ \widetilde{G_{1}}$ is defined with respect to $(\widetilde{\mathcal{G}}, \widetilde{\mathcal{E}})$.

\section{A Logic of LAYEREd GraphS}

We define a logical language, LGL, for expressing layering properties of graphs, and give an interpretation that uses the layered graph composition of the previous section. A different logic for graphs, with a separating (but associative and commutative) conjunction has also been explored in $[9,16]$.

Assume a set Atoms of atomic propositions, ranged over by p. The set, Formulae, of all propositional formulae is generated by the following grammar:

$$
\phi::=\mathrm{p}|\top| \perp|\phi \wedge \phi| \phi \vee \phi|\phi \rightarrow \phi| \phi \triangleright \phi|\phi \rightarrow \phi| \phi \smile \phi .
$$

The connectives above are the standard (classical additive) logical connectives, together with multiplicative conjunction, $\boldsymbol{\sim}$, and implications $\rightarrow$, We define $\neg \phi$ as $\phi \rightarrow \perp$.

A Hilbert-type logical calculus, $\mathbf{L} \mathbf{G L}_{\mathrm{H}}$, is given by the system of axioms and rules in Table 1. This system is closely related to the logic known as Boolean BI (BBI) [41] that features a commutative multiplicative fragment and an additive fragment with classical negation.

Proposition 3.1. The following rule is derivable in $\mathbf{L} \mathbf{G L}_{\mathrm{H}}$ :

$$
\frac{\eta \vdash \phi \quad \phi \vdash \psi}{\eta \vdash \psi} \quad \text { (Cut). }
$$

The proof is a short derivation using rules 11, 9 and then 10. Further structural properties (for example, idempotence, commutativity and unit laws) for the additive conjunction, $\wedge$, and unit, $\top)$ are also derivable. 

1. $\phi \vdash \phi$
2. $\phi \vdash \top$
3. $\quad \perp \vdash \phi$
4. $(\phi \rightarrow \perp) \rightarrow \perp \vdash \phi$
5. $\frac{\eta \vdash \phi \quad \eta \vdash \psi}{\eta \vdash \phi \wedge \psi}$
6. $\frac{\phi \vdash \psi_{1} \wedge \psi_{2}}{\phi \vdash \psi_{i}} \quad(i=1,2)$
7. $\frac{\eta \vdash \psi \quad \phi \vdash \psi}{\eta \vee \phi \vdash \psi}$
8. $\frac{\phi \vdash \psi_{i}}{\phi \vdash \psi_{1} \vee \psi_{2}} \quad(i=1,2)$
9. $\frac{\eta \wedge \phi \vdash \psi}{\eta \vdash \phi \rightarrow \psi}$
10. $\frac{\eta \vdash \phi \rightarrow \psi \quad \eta \vdash \phi}{\eta \vdash \psi}$
11. $\frac{\phi \vdash \psi}{\eta \wedge \phi \vdash \psi}$
12. $\frac{\xi \vdash \phi \quad \eta \vdash \psi}{\xi \triangleright \eta \vdash \phi \vdash \psi}$
13. $\frac{\eta \vdash \phi \vdash \psi}{\eta \vdash \phi \rightarrow \psi}$
14. $\frac{\xi \vdash \phi \rightarrow \psi \quad \eta \vdash \phi}{\xi \triangleright \eta \vdash \psi}$
15. $\frac{\eta \vdash \phi \vdash \psi}{\phi \vdash \eta \vdash \psi}$
16. $\frac{\xi \vdash \phi \vdash \psi \quad \eta \vdash \phi}{\eta \triangleright \xi \vdash \psi}$

TABLE 1. The Hilbert-type Logical Calculus $\mathbf{L G L}_{\mathrm{H}}$

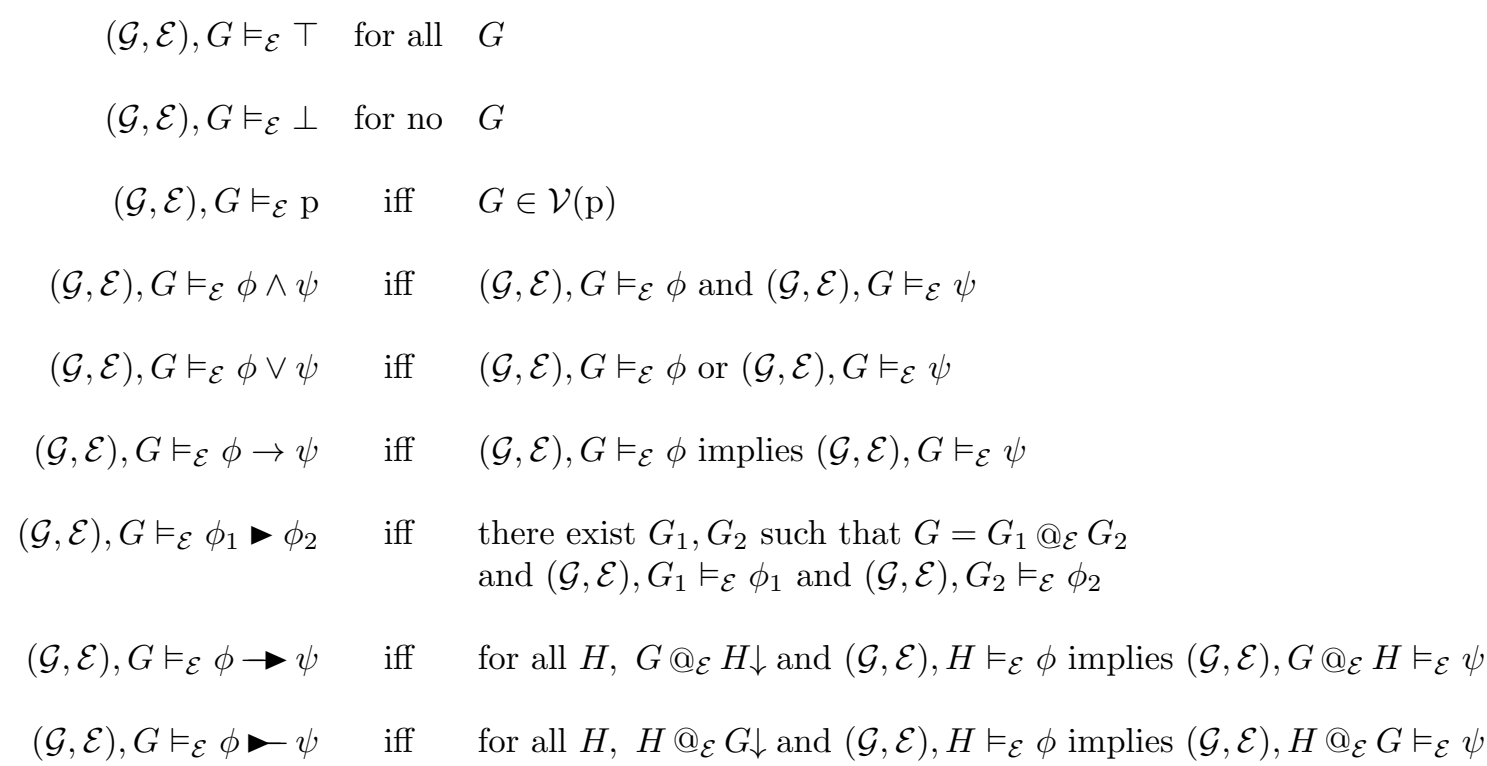

TABLE 2. The Satisfaction Relation for LGL

Given a scaffold $(\mathcal{G}, \mathcal{E})$ and a valuation $\mathcal{V}$ : Atoms $\longrightarrow \mathcal{P}(\operatorname{Sg}(\mathcal{G})$ ), where $\mathcal{P}$ is the powerset operator, the language can be given a semantics on the set of subgraphs of $\mathcal{G}$. The satisfaction relation is $\vDash_{\mathcal{E}} \subseteq \operatorname{Sg}(\mathcal{G}) \times$ Formulae. The definition of the satisfaction relation is given in Table 2 . Note that the clauses for $\rightarrow$, $\rightarrow$, and - make use of the graph composition $@_{\mathcal{E}}$, and that this is 
specific to $(\mathcal{G}, \mathcal{E})$.

Let $\llbracket \phi \rrbracket=\left\{G \mid G \vDash_{\mathcal{E}} \phi\right\}$, for every proposition $\phi$. This defines an interpretation function $\llbracket-\rrbracket:$ Formulae $\longrightarrow \mathcal{P}(\operatorname{Sg}(\mathcal{G}))$. Again, this is all relative to $(\mathcal{G}, \mathcal{E})$ and $\mathcal{V}$, and we suppress these in the notation for $\llbracket \phi \rrbracket$ when they are clear from the context. With this definition, it is easy to check that the following properties hold for all $\phi, \psi$, and $\xi$ :

$$
\begin{aligned}
& \llbracket \phi \wedge \psi \rrbracket \subseteq \llbracket \xi \rrbracket \quad \text { iff } \quad \llbracket \phi \rrbracket \subseteq \llbracket \psi \rightarrow \xi \rrbracket \\
& \llbracket \phi \triangleright \psi \rrbracket \subseteq \llbracket \xi \rrbracket \quad \text { iff } \quad \llbracket \phi \rrbracket \subseteq \llbracket \psi \rightarrow \xi \rrbracket \quad \text { iff } \quad \llbracket \psi \rrbracket \subseteq \llbracket \phi\llcorner\xi \rrbracket .
\end{aligned}
$$

These relationships underpin the adjointness relations for the implications $\rightarrow, \rightarrow$ and $\bullet$. We explore generalizations of this form of interpretation in Section 8.

Fix a scaffold $(\mathcal{G}, \mathcal{E})$, and consider an instance of the satisfaction relation of the form $(\mathcal{G}, \mathcal{E}), G \vDash_{\mathcal{E}}$ $\phi_{1} \downarrow \phi_{2}$, where $G$ is a subgraph of $\mathcal{G}$. This means that $G$ can be decomposed into subgraphs $G_{1}$ and $G_{2}$, that is $G=G_{1} @_{\mathcal{E}} G_{2}$, such that $G_{1}$ satisfies $\phi_{1}$ and $G_{2}$ satisfies $\phi_{2}$. The asymmetry of the composition operator, with edges from the component $G_{1}$ of $G$ to the component $G_{2}$, means that it is reasonable to regard $G_{1}$ as 'layered over' $G_{2}$, with respect to the ambient structure $(\mathcal{G}, \mathcal{E})$. This leads to the following logical characterization of what it means for one subgraph to be layered over another.

Let $G$ be a subgraph of a given scaffold $(\mathcal{G}, \mathcal{E})$. If $(\mathcal{G}, \mathcal{E}), G \vDash_{\mathcal{E}} \top \triangleright \top$, then we can also express the fact that $G$ is layered (with respect to $(\mathcal{G}, \mathcal{E})$ ) as a logical formula. Note that $G$ is layered if and only if there are $G_{1}$ and $G_{2}$ with $G=G_{1} @_{\mathcal{E}} G_{2}$. If $G_{1}$ and $G_{2}$ are any two such witnesses, then we say that $G_{1}$ is layered over $G_{2}$.

This definition allows there to be more than one layering of a particular subgraph over others within one ambient scaffold. For example, suppose that $V(\mathcal{G})=\left\{v_{1}, v_{2}, v_{3}\right\}, V\left(G_{i}\right)=\left\{v_{i}\right\}$ for $i=1,2,3, \mathcal{E}$ consists of an edge from $v_{1}$ to $v_{2}$ and an edge from $v_{1}$ to $v_{3}$, and $E(\mathcal{G})=\mathcal{E}$. In this case, we have both $G_{1} @_{\mathcal{E}} G_{2}$ and $G_{1} @_{\mathcal{E}} G_{3}$ defined.

Let $(\mathcal{G}, \mathcal{E})$ be an arbitrary scaffold, and let $G_{1}$ and $G_{2}$ be a particular pair of subgraphs. Let $\phi_{1}$ be an atomic proposition valued such that $(\mathcal{G}, \mathcal{E}), G \vDash_{\mathcal{E}} \phi_{1}$ iff $V(G)=V\left(G_{1}\right)$, and let $\phi_{2}$ be an atomic proposition valued such that $(\mathcal{G}, \mathcal{E}), G \vDash_{\mathcal{E}} \phi_{2}$ iff $V(G)=V\left(G_{2}\right)$, for all subgraphs $G$ of $\mathcal{G}$. Then $\llbracket \phi_{1}>\phi_{2} \rrbracket \subseteq \llbracket \neg\left(\phi_{2}>\phi_{1}\right) \rrbracket$ because of the contra-commutativity of @.

\section{Algebraic Structures and Interpretations}

The algebraic semantics of LGL has much in common with the approaches taken in [33, 4, 17, 18, 41, 20]. For brevity, where possible, we use the same name for mathematical structures and their carrier sets. If $\circ$ is any (partial), binary operation with an infix notation, then, for any element $x$ of the corresponding carrier set, we write $-\circ x$ and $x \circ-$ for the evident unary operations formed by fixing one argument with the particular element $x$.

A layered algebra is a structure $(\mathbb{A}, \wedge, \neg, \top, \boldsymbol{\longrightarrow} \rightarrow \longrightarrow)$, where $\mathbb{A}$ is the carrier set. The letters $A, B, C$ are used to range over the elements of $\mathbb{A}$. The operations $\wedge, \neg$, and $\top$ are required to define a Boolean algebra on $\mathbb{A}$, and are the standard notations for meet (binary infimum), Boolean complement, and $\top$ (the global supremum), respectively, with the operations $\vee, \rightarrow$ and $\perp$ defined in the usual way. The partial order $\leq$ on $\mathbb{A}$ is defined as usual by: $A \leq B$ iff $A=A \wedge B$, for all $A, B \in \mathbb{A}$.

The evident variant of the logic with intuitionistic additives would be defined by replacing the requirement that the operations $\wedge, \neg$, and $\top$ define a Boolean algebra on $\mathbb{A}$ with the requirement that a set of operations $\wedge, \vee, \top, \perp$, and $\rightarrow$ form a Heyting algebra on $\mathbb{A}$.

The symbols $\triangleright, \rightarrow$, and $\downarrow$ stand for binary operations. These operations are required to satisfy the following axioms, for all $A, A^{\prime}, B, B^{\prime}, C$ :

- If $A \leq A^{\prime}$ and $B \leq B^{\prime}$, then $A \triangleright B \leq A^{\prime}-B^{\prime}$;

- $A \triangleright B \leq C$ iff $B \leq A \rightarrow C$ iff $A \leq B-C$.

These properties can be stated equivalently in category-theoretic language, as follows:

- The operation - is a bifunctor;

- $A \rightarrow-$ is right-adjoint to the functor $A \triangleright-$ for all $A \in \mathbb{A}$; 
- $A \longrightarrow$ - is right-adjoint to the functor $\rightarrow A$ for all $A \in \mathbb{A}$.

This gives a pair of distinguished monoidal closed structures on the poset (regarded as a category).

Adapting terminology from $[17,18]$, a layered magma is a structure $(\mathbb{M}, \bullet)$ with a partial binary operation $\bullet$ on a carrier set $\mathbb{M}$. The operation is said to be contra-commutative if, for all $m, n \in \mathbb{M}$, if $m \bullet n$ is defined then $n \bullet m$ is undefined.

Proposition 4.1. Every scaffold $(\mathcal{G}, \mathcal{E})$ gives rise to a layered magma $\mathcal{M}(\mathcal{G}, \mathcal{E})$. In this case, the distinguished operation $\bullet$ is contra-commutative.

Proof. The carrier set of $\mathcal{M}(\mathcal{G}, \mathcal{E})$ is taken to be $\operatorname{Sg}(\mathcal{G})$. The distinguished operation is defined by $G \bullet H \simeq G @ H$, for all $G, H \in \operatorname{Sg}(\mathcal{G})$. The contra-commutativity property for the operation @ was noted in Proposition 2.3.

Proposition 4.2. Every layered magma $\mathbb{M}$ generates a layered algebra $\mathcal{A}(\mathbb{M})$.

Proof. The carrier set of $\mathcal{A}(\mathbb{M})$ is taken to be the powerset of the carrier of $\mathbb{M}$. The bifunctor is defined for all subsets $A$ and $B$ of $\mathbb{M}$ by $A \triangleright B=\{a \bullet b \mid a \in A, b \in B, a \bullet b$ is defined $\}$. Since powersets are always complete Boolean algebras, the rest of the structure is immediate; in particular, the existence of the adjoint functors $A \rightarrow-$ and $A \backsim-$ for all $A \in \mathbb{M}$.

In particular,

$$
\begin{aligned}
& A \rightarrow B=\{m \in \mathbb{M} \mid \forall a \in A, \text { if } m \bullet a \text { is defined, then } m \bullet a \in B\} \\
& A \vdash B=\{m \in \mathbb{M} \mid \forall a \in A, \text { if } a \bullet m \text { is defined, then } a \bullet m \in B\},
\end{aligned}
$$

for all subsets $A$ and $B$ of $\mathbb{M}$.

A valuation (for the logical language of Section 3) is a function $\mathcal{V}:$ Atoms $\longrightarrow \mathbb{A}$, where $\mathbb{A}$ is a layered algebra. Every valuation in $\mathbb{A}$ extends uniquely to an interpretation $\llbracket-\rrbracket:$ Formulae $\longrightarrow \mathbb{A}$ in the standard way, that is, by taking it to respect the connectives of the language. In particular, we have $\llbracket \phi \triangleright \psi \rrbracket=\llbracket \phi \rrbracket \triangleright \llbracket \psi \rrbracket, \llbracket \phi \rightarrow \psi \rrbracket=\llbracket \phi \rrbracket \rightarrow \llbracket \psi \rrbracket$, and $\llbracket \phi \neg \psi \rrbracket=\llbracket \phi \rrbracket-\llbracket \psi \rrbracket$.

It follows from Propositions 4.1 and 4.2 that we may interpret the logical language on both graphs and layered magmas: for a layered magma, $\mathbb{M}$, and valuation $\mathcal{V}:$ Formulae $\longrightarrow \mathcal{A}(\mathbb{M})$ we can consider $\llbracket-\rrbracket:$ Formulae $\longrightarrow \mathcal{A}(\mathbb{M})$; for a scaffold $(\mathcal{G}, \mathcal{E})$ and valuation $\mathcal{V}:$ Formulae $\longrightarrow$ $\mathcal{A}(\mathcal{M}(\mathcal{G}, \mathcal{E}))$, one has an interpretation $\llbracket-\rrbracket:$ Formulae $\longrightarrow \mathcal{A}(\mathcal{M}(\mathcal{G}, \mathcal{E}))$.

In the case of the interpretation on graphs, we recover the satisfaction definition of Section 3 , using the same valuation to define both: for every subgraph $G$ of the given ambient graph $\mathcal{G}$, and every formula $\phi$, we have that $(\mathcal{G}, \mathcal{E}), G \vDash_{\mathcal{E}} \phi$ iff $G \in \llbracket \phi \rrbracket$, agreeing with our earlier notation.

\section{Bi-LAYERING AND Stacking}

The notion of layering from above encapsulates an idea of irreversible connectivity from one zone (layer) to another. However, there are many situations in which zones are recognized as distinct, but one wants connectivity in both directions whilst recognizing the connections in each direction as different. In order to study such examples, we now introduce a new graph composition operator.

Let $\mathcal{G}$ be a graph and let $\langle\mathcal{E}, \mathcal{F}\rangle$ be an ordered pair of finite sets of edges such that $\mathcal{E}$ and $\mathcal{F}$ are disjoint. The bi-composition operator, $\hat{\Theta}_{\mathcal{E}, \mathcal{F}}$, is a partial, binary operator on the $\operatorname{set} \operatorname{Sg}(\mathcal{G})$. We omit the subscripts on the operator where the distinguished sets of edges $\mathcal{E}$ and $\mathcal{F}$ are unambiguous. The ambient graph $\mathcal{G}$ will always be clear from the context. For all $G_{1}, G_{2} \in \operatorname{Sg}(\mathcal{G})$, the expression $G_{1} \hat{@} G_{2}$ is defined just when both $G_{1} @_{\mathcal{E}} G_{2}$ and $G_{2} @_{\mathcal{F}} G_{1}$ are both defined. When $G_{1} @ G_{2}$ is defined, it is given by the vertex and edge sets

$$
V\left(G_{1} \hat{@} G_{2}\right)=V\left(G_{1}\right) \cup V\left(G_{2}\right) \quad \text { and } \quad E\left(G_{1} \hat{@} G_{2}\right)=E\left(G_{1}\right) \cup E\left(G_{2}\right) \cup\left((\mathcal{E} \cup \mathcal{F}) \cap \mathcal{G}\left[G_{1}, G_{2}\right]\right) \text {. }
$$

We have a bi-scaffold $(\mathcal{G},\langle\mathcal{E}, \mathcal{F}\rangle)$ if there is at least one pair of sub-graphs $\left(G_{1}, G_{2}\right)$ such that $G_{1} \hat{@} G_{2}$ is defined. 
Example 5.1. This example extends that of Example 2.1. Let $1 \leq k \leq m, n, G_{1}, G_{2}$ and $\mathcal{E}$ be as before, but in addition require that $m \geq 2 k$. Consider the set $T=\left\{\left(w_{i}, v_{2 i}\right) \mid 1 \leq i \leq k\right\}$, and let $\mathcal{F}$ be the set of directed edges given by $T$. Then $G_{1} \hat{\Theta}_{\mathcal{E}, \mathcal{F}} G_{2}$ is defined, where $\mathcal{G}$ is defined by the same vertex and edge sets as $G_{1} \widehat{@}_{\mathcal{E}, \mathcal{F}} G_{2}$. Figure 5 illustrates this example, again with $m=5, n=4$.
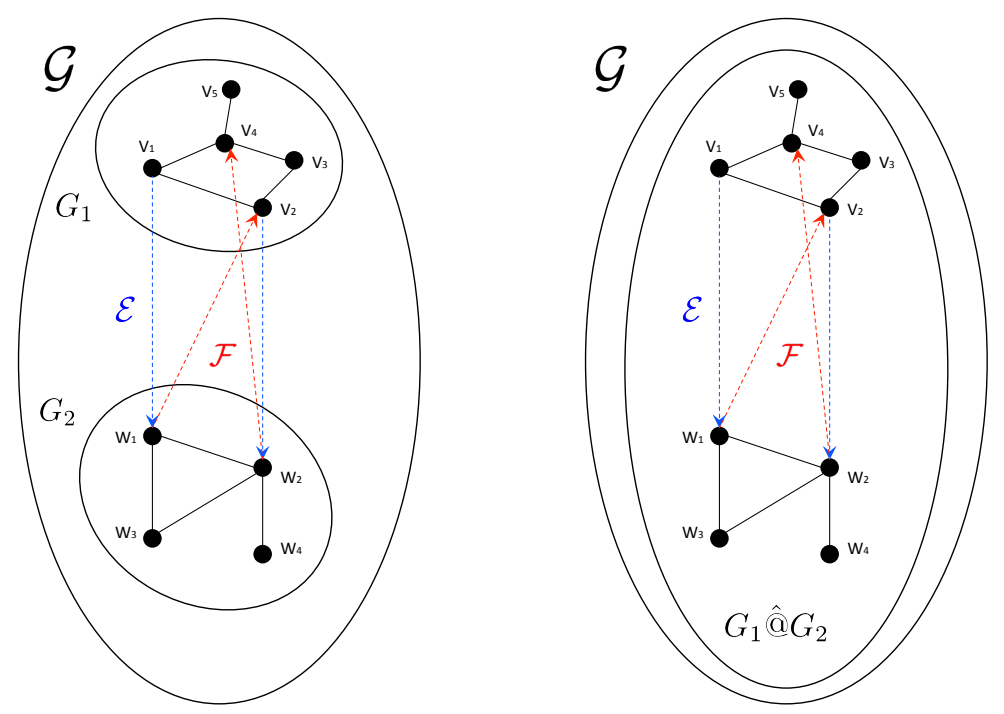

Figure 5. Bi-Layer Construction of Example 5.1

The operator @ is not necessarily associative (up to Kleene-equality) because of the definedness conditions on the operator.

Example 5.2. Let $V\left(G_{i}\right)=\left\{v_{i}\right\}$ for $i=1,2,3$. Consider the sets of edges defined by the sets of ordered pairs $\mathcal{E}=\left\{\left(v_{1}, v_{2}\right),\left(v_{1}, v_{3}\right)\right\}, \mathcal{F}=\left\{\left(v_{2}, v_{1}\right),\left(v_{3}, v_{2}\right)\right\}$. Let $V(\mathcal{G})=\left\{v_{1}, v_{2}, v_{3}\right\}$ and $E(\mathcal{G})=\mathcal{E} \cup \mathcal{F}$. Then $\left(G_{1} \hat{\Theta}_{\mathcal{E}, \mathcal{F}} G_{2}\right) \hat{\Theta}_{\mathcal{E}, \mathcal{F}} G_{3}$ is defined, but $G_{1} \hat{\mathrm{\Theta}}_{\mathcal{E}, \mathcal{F}}\left(G_{2} \hat{\mathrm{@}}_{\mathcal{E}, \mathcal{F}} G_{3}\right)$ is not.

Let $G_{1}, G_{2}$ be subgraphs of some bi-scaffold $(\mathcal{G},\langle\mathcal{E}, \mathcal{F}\rangle)$. If $G_{1} @_{\mathcal{E}} G_{2}$ and $G_{2} @_{\mathcal{F}} G_{1}$ are defined, then $G_{1} \hat{@} G_{2}$ is bi-layered with respect to $(\mathcal{G},\langle\mathcal{E}, \mathcal{F}\rangle)$.

For a fixed graph $\mathcal{G}$, and disjoint edge sets $\mathcal{E}, \mathcal{F}$ observe that if $\mathcal{E}=\emptyset$ or $\mathcal{F}=\emptyset$ then there are no bi-layered subgraphs and $(\mathcal{G},\langle\mathcal{E}, \mathcal{F}\rangle)$ is not a bi-scaffold. There are also many examples in which both $(\mathcal{G}, \mathcal{E})$ and $(\mathcal{G}, \mathcal{F})$ are scaffolds but $(\mathcal{G},\langle\mathcal{E}, \mathcal{F}\rangle)$ is not a bi-scaffold.

Example 5.3. Let $V(\mathcal{G})=\left\{v_{1}, v_{2}, v_{3}, v_{4}\right\}$. Let $\mathcal{E}=\left\{\left(v_{1}, v_{2}\right),\left(v_{3}, v_{1}\right)\right\}$ and $\mathcal{F}=\left\{\left(v_{3}, v_{4}\right)\right\}$, and let $E(\mathcal{G})=\mathcal{E} \cup \mathcal{F}$. Let $G_{i}$ be the single vertex $v_{i}$ for each $i=1,2,3,4$. Then $G_{1} @_{\mathcal{E}} G_{2}$ and $G_{3} @_{\mathcal{F}} G_{4}$ are both defined, but there are no subgraphs $G, H$ of $\mathcal{G}$ with $G \hat{@}_{\mathcal{E}, \mathcal{F}} H$ defined.

So far we have studied only the construction and decomposition of layered structures with exactly two layers. However, layered structures occurring in examples often consist of more than two layers. Unfortunately, the failure of associativity of both @ and @ constrains their applicability when it comes to describing situations with multiple layers. The following proposition highlights these constraints, motivating the need for the n-ary composite immediately after it. Such a composite allows instances of multiple layers to be explored.

Proposition 5.4. Let $(\mathcal{G},\langle\mathcal{E}, \mathcal{F}\rangle)$ be a bi-scaffold, and $G_{1}, G_{2}$ be subgraphs of $\mathcal{G}$. Then:

(1) Both $(\mathcal{G}, \mathcal{E})$ and $(\mathcal{G}, \mathcal{F})$ are scaffolds;

(2) The bi-composition operator $\hat{\Theta}_{\mathcal{E}, \mathcal{F}}$ is contra-commutative; that is, if $G_{1} \hat{\Theta}_{\mathcal{E}, \mathcal{F}} G_{2}$ is defined, then $G_{2} \hat{@}_{\mathcal{E}, \mathcal{F}} G_{1}$ is not;

(3) $G_{1} \hat{\Theta}_{\mathcal{E}, \mathcal{F}} G_{1}$ is undefined;

(4) $(\mathcal{G},\langle\mathcal{F}, \mathcal{E}\rangle)$ is a bi-scaffold; 
(5) $G_{1} \hat{@}_{\mathcal{E}, \mathcal{F}} G_{2} \simeq G_{2} \hat{@}_{\mathcal{F}, \mathcal{E}} G_{1}$.

Let $n>1$, let $\mathcal{G}$ be a graph and $\mathcal{E}_{1}, \ldots, \mathcal{E}_{n-1}$ be non-empty sets of edges of $\mathcal{G}$. Let the strong composition @ $\left(G_{1}, \ldots, G_{n}\right)$ of any subgraphs $G_{1}, \ldots, G_{n}$ of $\mathcal{G}$ be defined just when $V\left(G_{i}\right) \cap$ $V\left(G_{j}\right)=\emptyset$ for all $i \neq j$ and $\mathcal{E}_{i} \subseteq \mathcal{G}\left(G_{i}, G_{i+1}\right)$ for all $i$; when it is defined, let

$$
\begin{gathered}
V\left(\underline{@}\left(G_{1}, \ldots, G_{n}\right)\right)=\bigcup_{1 \leq i \leq n} V\left(G_{i}\right) \quad \text { and } \\
E\left(\underline{@}\left(G_{1}, \ldots, G_{n}\right)\right)=\left(\bigcup_{1 \leq i \leq n} E\left(G_{i}\right)\right) \cup\left(\bigcup_{1 \leq i \leq n-1} \mathcal{E}_{i}\right) .
\end{gathered}
$$

In the case $n=1$, it is convenient to define $G=\underline{@}(G)$. Note that what is being defined here is an $n$-ary partial operator. We refer to such $n$-ary composites as (layered) stacks. Where the distinguished sets of edges need to be made clear, we use an infix notation of the form

$$
G_{1} \underline{\Theta}_{\mathcal{E}_{1}} G_{2} \underline{\Theta}_{\mathcal{E}_{2}} \cdots \underline{\mathcal{E}}_{\mathcal{E}_{n-2}} G_{n-1} \underline{@}_{\mathcal{E}_{n-1}} G_{n} .
$$

Examples of stacks arise in $[36,19,37]$.

Proposition 5.5. Let $\mathcal{G}$ be a graph, let all $G_{i}$ below be non-empty subgraphs of $\mathcal{G}$, and all $\mathcal{E}_{i}$ be non-empty sets of edges of $\mathcal{G}$.

1. Let $G_{1} \underline{\Theta}_{\mathcal{E}_{1}} \ldots \underline{@}_{\mathcal{E}_{n-1}} G_{n}$ be defined. Then $G_{k} \underline{\mathcal{E}}_{\mathcal{E}_{k}} \ldots \underline{\mathcal{E}}_{\mathcal{E}_{m-1}} G_{m}$ is defined, where $1 \leq k \leq$ $m \leq n$. That is, if an $n$-ary strong composite is defined with respect to a sequence of $n-1$ edge sets, then the evident $(1+m-k)$-ary strong composite is defined for any consecutive subsequence. In particular, this holds for $m=k+1$, so that $G_{k} \underline{\mathcal{E}}_{\mathcal{E}_{k}} G_{k+1}$ is defined. Moreover,

$$
\begin{aligned}
& G_{1} \underline{\mathcal{E}}_{\mathcal{E}_{1}} \ldots \underline{\mathcal{E}}_{\mathcal{E}_{n-1}} G_{n}=
\end{aligned}
$$

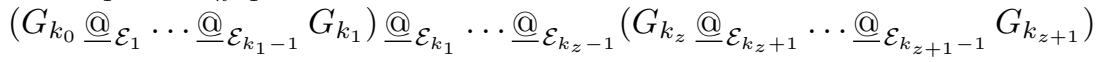

for all $1=k_{0} \leq k_{1} \leq \ldots \leq k_{z} \leq k_{z+1}=n$ and $0 \leq z \leq n$ such that the right-handside 'partitions' the sequence from the left-hand-side into consecutive subsequences (each expression in brackets). Each of the bracketed expressions $G_{k_{i}} \underline{\varrho}_{\mathcal{E}_{i}+1} \ldots \underline{\mathcal{E}}_{\mathcal{E}_{k_{i+1}-1}} G_{k_{i+1}}$ on the right-hand-side is intended to be $a\left(1+k_{i+1}-k_{i}\right)$-ary composite.

2. Let $1=k_{0} \leq k_{1} \leq \ldots \leq k_{z} \leq k_{z+1}=n$, where $0 \leq z \leq n$. Let $V\left(G_{1}\right), \ldots, V\left(G_{n}\right)$ be a pairwise disjoint family of vertices, and $\mathcal{E}_{1}, \ldots, \mathcal{E}_{n-1}$ be a pairwise disjoint family of nonempty sets of edges. If the composite $G_{k_{i}} \underline{\Theta}_{\mathcal{E}_{k_{i}}} \ldots \underline{\mathcal{E}}_{\mathcal{E}_{k_{i+1}-1}} G_{k_{i+1}}$ is defined for all even $0 \leq i \leq z$, and $k_{i}<k_{i+1}=k_{i}+1$ and $\mathcal{E}_{k_{i}} \subseteq \mathcal{G}\left(G_{k_{i}}, G_{k_{i}+1}\right)$ for all odd $0 \leq i \leq z$, then $G_{1} @_{\mathcal{E}_{1}} \ldots \underline{@}_{\mathcal{E}_{n-1}} G_{n}$ is defined, and Equation (2) holds.

3. If $G_{1} \underline{\mathcal{E}}_{\mathcal{E}_{1}} G_{2}$ is defined, then $G_{1} @_{\mathcal{E}_{1}} G_{2}$ is defined, and

$$
G_{1} @_{\mathcal{E}_{1}} G_{2}=G_{1} @_{\mathcal{E}_{1}} G_{2} \text {. }
$$

4. Let $G_{1}, G_{2}, G_{3}$ be subgraphs of $G$. If $G_{1} @_{\mathcal{E}_{1}} G_{2} @_{\mathcal{E}_{2}} G_{3}$ is defined, then $G_{1} @_{\mathcal{E}_{1}}\left(G_{2} @_{\mathcal{E}_{2}} G_{3}\right)$ and $\left(G_{1} @_{\mathcal{E}_{1}} G_{2}\right) @_{\mathcal{E}_{2}} G_{3}$ are both defined, and

$$
G_{1} @_{\mathcal{E}_{1}} G_{2}{\stackrel{@}{\mathcal{E}_{2}}} G_{3}=G_{1} @_{\mathcal{E}_{1}}\left(G_{2} @_{\mathcal{E}_{2}} G_{3}\right)=\left(G_{1} @_{\mathcal{E}_{1}} G_{2}\right) @_{\mathcal{E}_{2}} G_{3} .
$$

Proof. All points of the proposition are more-or-less immediate consequences of the definitions. Only the first point is explained in more detail below:

1. Suppose that $G_{1} \underline{\mathcal{E}}_{1} \ldots \underline{\mathcal{E}}_{\mathcal{E}_{n-1}} G_{n}$ is defined. Let $G_{k} \underline{\mathcal{E}}_{\mathcal{E}_{k}} \ldots \underline{\mathrm{E}}_{\mathcal{E}_{m-1}} G_{m}$ with $1 \leq k \leq$ $m \leq n$ be a composition of a consecutive subsequence, and note that this must be defined. All of the conditions are satisfied in order for the composite of the right-hand-side of Equation (2) to be defined. Suppose that $1=k_{0} \leq k_{1} \leq \ldots \leq k_{z} \leq k_{z+1}=n$, where $0 \leq z \leq n$. As above, $G_{k_{i}} \underline{\mathcal{E}}_{k_{k_{i}+1}} \ldots \underline{@}_{\mathcal{E}_{k_{i+1}-1}} G_{k_{i+1}}$ is defined for all $0 \leq i \leq z$. Furthermore, all of the edges of $\mathcal{E}_{k_{i}+1}$ begin in $G_{k_{i}} \underline{\varrho}_{\mathcal{E}_{k_{i}+1}} \ldots \underline{\mathcal{E}}_{\mathcal{E}_{k_{i+1}-1}} G_{k_{i+1}}$ and end in $G_{k_{i+1}} \underline{\mathcal{E}}_{\mathcal{k}_{i+1}+1} \ldots \underline{\varrho}_{\mathcal{E}_{k_{i+2}-1}} G_{k_{i+2}}$, so the composite on the right-hand-side of Equation 2 is defined. The union of the graphs and edge-sets 
defined by the composite on the left-hand-side of Equation (2) is then evidently identical to the union of the graphs and edge-sets defined by the composite on the right-hand-side.

In a similar way, we can also consider the formation of bi-layered stacks via $n$-ary partial composition operations. Let $\mathcal{G}$ be a graph and $\mathcal{E}_{1}, \ldots, \mathcal{E}_{n-1}, \mathcal{F}_{1}, \ldots, \mathcal{F}_{n-1}$ be non-empty sets of edges of $\mathcal{G}$. Let the strong bi-composition $\underline{\hat{@}}\left(G_{1}, \ldots, G_{n}\right)$ of any subgraphs $G_{1}, \ldots, G_{n}$ of $\mathcal{G}$ be defined just when $V\left(G_{i}\right) \cap V\left(G_{j}\right)=\emptyset$ for all $i \neq j$, and $\mathcal{E}_{i} \subseteq \mathcal{G}\left(G_{i}, G_{i+1}\right)$ and $\mathcal{F}_{i} \subseteq \mathcal{G}\left(G_{i+1}, G_{i}\right)$ for all $i$; when it is defined, let

$$
\begin{gathered}
V\left(\underline{\hat{\mathrm{a}}}\left(G_{1}, \ldots, G_{n}\right)\right)=\bigcup_{1 \leq i \leq n} V\left(G_{i}\right) \quad \text { and } \\
E\left(\underline{\hat{\mathrm{a}}}\left(G_{1}, \ldots, G_{n}\right)\right)=\left(\bigcup_{1 \leq i \leq n} E\left(G_{i}\right)\right) \cup\left(\bigcup_{1 \leq i \leq n-1} \mathcal{E}_{i} \cup \mathcal{F}_{i}\right) .
\end{gathered}
$$

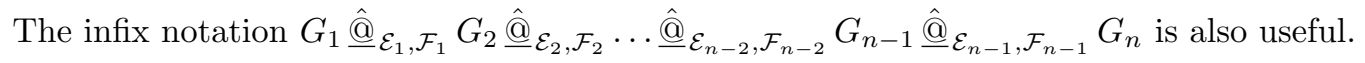

Proposition 5.6. Consider a sequence of subgraphs $G_{1}, \ldots, G_{n}$ of $\mathcal{G}$, and sequences of edges $\mathcal{E}_{1}, \ldots, \mathcal{E}_{n-1}$ and $\mathcal{F}_{1}, \ldots \mathcal{F}_{n-1}$ of $\mathcal{G}$. If $\mathcal{E}_{i} \cap \mathcal{F}_{j}=\emptyset$, for all $1 \leq i, j<n$, then

$$
G_{1} \underline{\hat{@}}_{\mathcal{E}_{1}, \mathcal{F}_{1}} \ldots \underline{\hat{@}}_{\mathcal{E}_{n-1}, \mathcal{F}_{n-1}} G_{n} \simeq\left(G_{1} \underline{@}_{\mathcal{E}_{1}} \ldots \underline{\underline{@}}_{\mathcal{E}_{n-1}} G_{n}\right) \cup\left(G_{n} \underline{@}_{\mathcal{F}_{n-1}} \ldots \underline{@}_{\mathcal{F}_{1}} G_{1}\right)
$$

where $\cup$ is the usual union of graphs.

Proof. If the composites on either side are defined, then they have the same sets of edges and vertices, and so are equal. The strong bi-composition on the left-hand-side is defined precisely when both of the strong compositions on the right-hand-side are defined.

Proposition 5.7. A bi-layered graph is a special case of a bi-layered stack.

Proof. The proof is an immediate corollary of Propositions 5.5(3) and 5.6.

Stacks with $n>2$ layers seem to be strong compositions in an essential way, rather than just iterated binary compositions. An iterated binary composition would not capture the notion of sequential edge sets joining layers; rather, it could lead to a large collection of inter-connected subgraphs with little discernible structure.

One can give logical characterizations of the notions of stacking and bi-layering explored in this section that generalize the logical characterization of Section 3. This is discussed in the next section and also leads to interesting results based on logical satisfaction with respect to differing edge sets.

\section{Logical Characterizations of Bi-Layering and Stacking}

One can define a notion of graph-interpretation of logical formulae for the same language LGL, but now using the bi-composition operator $\hat{\mathfrak{\Theta}}_{\mathcal{E}, \mathcal{F}}$ to give a magma on the set of subgraphs of a given bi-scaffold $(\mathcal{G},\langle\mathcal{E}, \mathcal{F}\rangle)$. This now makes LGL into a language for describing properties of bi-layered graphs under the new interpretation.

For a given valuation $\mathcal{V}:$ Atoms $\longrightarrow \mathcal{P}(\operatorname{Sg}(\mathcal{G}))$, let the resulting interpretation be $\llbracket-\rrbracket_{\mathcal{E}, \mathcal{F}}:$

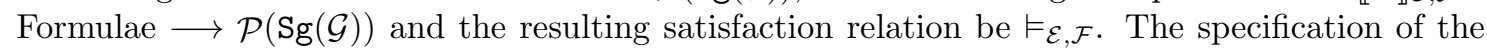
new satisfaction relation has the same form as that given in Table 2, but uses the operator $\hat{@}_{\mathcal{E}, \mathcal{F}}$ in place of @ $\mathcal{E}$. For example,

$$
\begin{aligned}
(\mathcal{G},\langle\mathcal{E}, \mathcal{F}\rangle), G \vDash_{\mathcal{E}, \mathcal{F}} \phi \downarrow \psi \quad \text { iff } & \text { there exist } G_{1}, G_{2} \text { such that } G=G_{1} \hat{\Theta}_{\mathcal{E}, \mathcal{F}} G_{2} \\
& \text { and }(\mathcal{G},\langle\mathcal{E}, \mathcal{F}\rangle), G_{1} \vDash_{\mathcal{E}, \mathcal{F}} \phi \text { and }(\mathcal{G},\langle\mathcal{E}, \mathcal{F}\rangle), G_{2} \vDash_{\mathcal{E}, \mathcal{F}} \psi .
\end{aligned}
$$

Also note that, for atomic propositions $\phi$ and $\psi,(\mathcal{G},\langle\mathcal{E}, \mathcal{F}\rangle), G \vDash_{\mathcal{E}, \mathcal{F}} \phi \downarrow \psi$ iff $(\mathcal{G},\langle\mathcal{F}, \mathcal{E}\rangle), G \vDash_{\mathcal{F}, \mathcal{E}}$ $\psi \triangleright \phi$.

Let $\llbracket-\rrbracket_{\mathcal{E}}$ and $\vDash_{\mathcal{E}}$ be the interpretation and satisfaction relation on the magma with composition $@_{\mathcal{E}}$, and let $\llbracket-\rrbracket_{\mathcal{F}}$ and $\vDash_{\mathcal{F}}$ similarly correspond to $@_{\mathcal{F}}$. 
The logical characterization for bi-layering is similar to that for layering: a subgraph $G$ is bi-layered with respect to the chosen edges sets $\mathcal{E}$ and $\mathcal{F}$ of $\mathcal{G}$ if $(\mathcal{G},\langle\mathcal{E}, \mathcal{F}\rangle), G \vDash_{\mathcal{E}, \mathcal{F}} \top \longrightarrow \top$.

If $G$ is bi-layered with respect to $\mathcal{E}$ and $\mathcal{F}$, then it is layered with respect to both $\mathcal{E}$ and $\mathcal{F}$ : if $(\mathcal{G},\langle\mathcal{E}, \mathcal{F}\rangle), G \vDash_{\mathcal{E}, \mathcal{F}} \top \triangleright T$, then $(\mathcal{G}, \mathcal{E}), G \vDash_{\mathcal{E}} \top \triangleright T$ and $(\mathcal{G}, \mathcal{F}), G \vDash_{\mathcal{F}} \top \triangleright \top$.

Example 6.1. Consider a graph $\mathcal{G}$ with precisely two vertices $v, w$ and two edges e, $f$ from $v$ to w. Let $\mathcal{E}=\{e\}$ and $\mathcal{F}=\{f\}$. Then, considered as a subgraph of itself, $(\mathcal{G}, \mathcal{E}), G \vDash_{\mathcal{E}} \top \backslash \top$ and $(\mathcal{G}, \mathcal{F}), G \vDash_{\mathcal{F}} \top \triangleright \top$, but $(\mathcal{G},\langle\mathcal{E}, \mathcal{F}\rangle), G \not \forall_{\mathcal{E}, \mathcal{F}} \top \triangleright \top$.

This shows that there are examples of formulae $\phi$ of interest with $\llbracket \phi \rrbracket_{\mathcal{E}} \nsubseteq \llbracket \phi \rrbracket_{\mathcal{E}, \mathcal{F}}$, for particular choices of ambient structure. The following proposition, which is an immediate consequence of the definitions, illustrates important aspects of the relationships between the interpretations:

Proposition 6.2. For all $\phi$ and $\psi$, the following hold:

(1) If $\llbracket \phi \rrbracket_{\mathcal{E}, \mathcal{F}} \subseteq \llbracket \phi \rrbracket_{\mathcal{E}}$ and $\llbracket \psi \rrbracket_{\mathcal{E}, \mathcal{F}} \subseteq \llbracket \psi \rrbracket_{\mathcal{E}}$, then $\llbracket \phi \triangleright \psi \rrbracket_{\mathcal{E}, \mathcal{F}} \subseteq \llbracket \phi \triangleright \psi \rrbracket_{\mathcal{E}} ;$

(2) If $\llbracket \phi \rrbracket_{\mathcal{E}, \mathcal{F}} \subseteq \llbracket \phi \rrbracket_{\mathcal{E}}$ and $\llbracket \psi \rrbracket_{\mathcal{E}} \subseteq \llbracket \psi \rrbracket_{\mathcal{E}, \mathcal{F}}$, then $\llbracket \phi \rightarrow \psi \rrbracket_{\mathcal{E}} \subseteq \llbracket \phi \rightarrow \psi \rrbracket_{\mathcal{E}, \mathcal{F}}$;

(3) If $\llbracket \phi \rrbracket_{\mathcal{E}} \subseteq \llbracket \phi \rrbracket_{\mathcal{E}, \mathcal{F}}$ and $\llbracket \psi \rrbracket_{\mathcal{E}, \mathcal{F}} \subseteq \llbracket \psi \rrbracket_{\mathcal{E}}$, then $\llbracket \phi \rightarrow \psi \rrbracket_{\mathcal{E}, \mathcal{F}} \subseteq \llbracket \phi \rightarrow \psi \rrbracket_{\mathcal{E}}$.

The contravariance in the second and third parts of the above proposition and examples such as that immediately below means that, even if the valuation of atomic propositions is identical for each of the interpretations $\llbracket-\rrbracket_{\mathcal{E}}, \llbracket-\rrbracket_{\mathcal{F}}$ and $\llbracket-\rrbracket_{\mathcal{E}, \mathcal{F}}$, the relationship between those interpretations is not simple. This is further illustrated by the following example, in which there is a formula $\psi$ with $\llbracket \psi \rrbracket \mathcal{E}, \mathcal{F} \nsubseteq \llbracket \psi \rrbracket_{\mathcal{E}}$.

Example 6.3. Let $\mathcal{G}$ be the same as in Example 6.1. Let $G$ be the subgraph of $\mathcal{G}$ with $V(G)=$ $\{v\}$. Let $\phi$ be the atomic proposition 'contains vertex $w$ ': more precisely, let the valuation (for all three interpretations $\left.\llbracket-\rrbracket_{\mathcal{E}}, \llbracket-\rrbracket_{\mathcal{F}}, \llbracket-\rrbracket_{\mathcal{E}, \mathcal{F}}\right)$ have $\mathcal{V}(\phi)=\{H \in \operatorname{Sg}(\mathcal{G}) \mid w \in V(H)\}$. Then $(\mathcal{G},\langle\mathcal{E}, \mathcal{F}\rangle), G \vDash_{\mathcal{E}, \mathcal{F}} \phi \rightarrow \perp$, because there are no $H$ with $G \hat{@}_{\mathcal{E}, \mathcal{F}} H$ defined and $(\mathcal{G},\langle\mathcal{E}, \mathcal{F}\rangle), H \vDash_{\mathcal{E}, \mathcal{F}}$ $\phi$. However, $(\mathcal{G}, \mathcal{E}), G \vDash_{\mathcal{E}} \phi \rightarrow \perp$ can be refuted by taking $H$ with $(\mathcal{G}, \mathcal{E}), H \vDash_{\mathcal{E}} \phi$ to be the subgraph with $V(H)=\{w\}$.

We now define another logical language, SGL, that is suited to describing properties of stacks. The connectives of SGL are the same as those of $\mathbf{L G L}$, except that $\boldsymbol{\longrightarrow} \rightarrow$ and $\downarrow$ are replaced by

- a family of $n$-ary connectives $\boldsymbol{}^{n}$, indexed by integers $n \geq 2$, and

- a family of $n$-ary connectives $\rightarrow^{n, m}$ indexed by integers $n \geq 2$ and $1 \leq m<n$.

A formula with principal connective $\rightarrow^{n, m}$ can be eliminated by constructing a formula $\boldsymbol{}^{n}$ in which the hypotheses for $\rightarrow{ }^{n, m}$ occur in the $m$ th place in $\boldsymbol{}^{n}$. Thus this set-up allows for the formation of new propositions

$$
>^{n}\left(\phi_{1}, \ldots, \phi_{n}\right) \quad \text { and } \quad \rightarrow^{n, m}\left(\phi_{1}, \ldots, \phi_{n}\right)
$$

given any sequence of propositions $\phi_{1}, \ldots, \phi_{n}$. Note that this defines a new set, Formulae , of logical formulae.

Define a new logical calculus $\mathbf{S G L}_{\mathrm{H}}$ by: taking axioms to apply to formulae of the language SGL; taking the rules of $\mathbf{L G L}_{\mathrm{H}}$ and removing the rules containing the connectives $\rightarrow$, $\rightarrow$ and $\checkmark$; adding the three rules shown in Table 3 . In rule (13s), $n \geq 1$ and $\eta$ lies at the $m$ th argument of ${ }^{n+1}\left(\phi_{1}, \ldots, \eta, \ldots, \phi_{n}\right)$. In rule $(14 \mathrm{~s}), n \geq 1$ and the $i$ th $\operatorname{argument}$ of $\boldsymbol{}^{n+1}\left(\xi_{1}, \ldots, \eta, \ldots, \xi_{n}\right)$ is $\xi_{i}$ if $1 \leq i<m, \eta$ if $i=m$, and $\xi_{i-1}$ if $m<i \leq n$.

A multi-layered algebra is a structure

$$
\mathbb{A}=\left(\mathbb{A}, \wedge, \neg, \top,\left(\boldsymbol{}^{n}\right)_{n \geq 2},\left(\rightarrow^{n, m}\right)_{n \geq 2,1 \leq m<n}\right)
$$

such that:

- $\mathbb{A}=(\mathbb{A}, \wedge, \neg, \top)$ defines a Boolean algebra (with $\vee, \perp, \rightarrow$ and partial order $\leq$ definable in the usual way);

- For each $n \geq 2$ the operator ${ }^{n}$ is $n$-ary, and order-preserving;

- For each $n \geq 2$ and $1 \leq m<n$, the operator $\rightarrow n, m$ is $n$-ary; 


$$
\begin{gathered}
\frac{\psi_{1} \vdash \phi_{1} \quad \ldots \quad \psi_{n} \vdash \phi_{n}}{\triangleright^{n}\left(\psi_{1}, \ldots, \psi_{n}\right) \vdash{ }^{n}\left(\phi_{1}, \ldots, \phi_{n}\right)} \\
\frac{\triangleright^{n+1}\left(\phi_{1}, \ldots, \eta, \ldots, \phi_{n}\right) \vdash \psi}{\eta \vdash \rightarrow{ }^{n+1, m}\left(\phi_{1}, \ldots, \phi_{n}, \psi\right)} \\
\frac{\eta \vdash \rightarrow{ }^{n+1, m}\left(\phi_{1}, \ldots, \phi_{n}, \psi\right) \quad \xi_{1} \vdash \phi_{1} \quad \ldots \quad \xi_{n} \vdash \phi_{n}}{\triangleright^{n+1}\left(\xi_{1}, \ldots, \eta, \ldots, \xi_{n}\right) \vdash \psi}
\end{gathered}
$$

TABLE 3. Multiplicative Rules for $\mathbf{S G L}_{\mathrm{H}}$

- For each $n \geq 2$ and $1 \leq m<n$,

$$
\boldsymbol{}^{n}\left(A_{1}, \ldots, A_{n}\right) \leq A_{n+1} \quad \text { iff } \quad A_{m} \leq \rightarrow^{n, m}\left(A_{1}, \ldots, A_{m-1}, A_{m}, \ldots, A_{n+1}\right)
$$

for all $A_{1}, \ldots, A_{n+1} \in \mathbb{A}$.

Note that for all $A_{1}, \ldots, A_{n} \in \mathbb{A}$ there is an order-preserving function

$$
\boldsymbol{\nabla}^{n}\left(A_{1}, \ldots, A_{m-1},-, A_{m+1}, \ldots, A_{n}\right): \mathbb{A} \longrightarrow \mathbb{A} \text {. }
$$

The conditions demand that the assignment

$$
A_{n+1} \mapsto \rightarrow^{n, m}\left(A_{1}, \ldots, A_{m-1}, A_{m+1}, \ldots, A_{n+1}\right)
$$

defines a right-adjoint to each functor $\nabla^{n}\left(A_{1}, \ldots, A_{m-1},-, A_{m+1}, \ldots, A_{n}\right)$.

Define a multi-magma to be a structure $\left(M,\left(\bullet^{n}\right)_{n \geq 2}\right)$ with a carrier set $M$, and a partial, $n$-ary operation $\bullet^{n}$ on $M$ for each integer $n \geq 2$.

Let $\mathcal{G}$ be a given graph. Let $\mathcal{E}_{1}, \mathcal{E}_{2}, \ldots$ be a pairwise disjoint sequence of sets of edges of $\mathcal{G}$. Let $M$ be the set of sub-graphs of $\mathcal{G}$. For each $n \geq 2$ and each sequence of graphs $G_{1}, \ldots, G_{n}$, define

$$
\bullet^{n}\left(G_{1}, \ldots, G_{n}\right) \simeq G_{1} \underline{\Theta}_{\mathcal{E}_{1}} \cdots \underline{\mathfrak{Q}}_{\mathcal{E}_{n-1}} G_{n} .
$$

Note that this is intended to be undefined if the right-hand-side is undefined, and that this is the case if any of $\mathcal{E}_{1}, \ldots, \mathcal{E}_{n-1}$ are empty. Thus strong-composition with respect to $\mathcal{E}_{1}, \mathcal{E}_{2}, \ldots$ on $\mathcal{G}$ gives a multi-magma. Similarly, one can define a multi-magma based on the strong bi-composition.

Proposition 6.4. Each multi-magma $M$ gives rise to a multi-layered algebra on the power-set of the carrier of $M$.

Proof. The construction is an easy generalization of the earlier, strictly binary case: define

$$
\boldsymbol{\triangleright}^{n}\left(A_{1}, \ldots, A_{n}\right)=\left\{\bullet^{n}\left(a_{1}, \ldots, a_{n}\right) \mid \forall 1 \leq i \leq n . a_{i} \in A_{i}\right\}
$$

for all $n \geq 2$ and $A_{1}, \ldots, A_{n} \subseteq M$. Each operation $\nabla^{n}$ is order-preserving and the adjointness conditions hold because of the completeness of the Boolean algebra on the power-set.

A valuation of atomic propositions in a layered algebra is a function $\mathcal{V}:$ Atoms $\longrightarrow \mathbb{A}$, as before. This then extends, suppressing subscripts, to an interpretation $\llbracket-\rrbracket:$ Formulae $_{S} \longrightarrow \mathbb{A}$ of all propositions in the standard way; in particular,

- $\triangleright^{n}\left(\phi_{1}, \ldots, \phi_{n}\right) \rrbracket=\boldsymbol{}^{n}\left(\llbracket \phi_{1} \rrbracket, \ldots, \llbracket \phi_{n} \rrbracket\right)$ and

- $\rightarrow^{n, m}\left(\phi_{1}, \ldots, \phi_{n}\right) \rrbracket=\rightarrow^{n, m}\left(\llbracket \phi_{1} \rrbracket, \ldots, \llbracket \phi_{n} \rrbracket\right)$.

The interpretation is sound - the proof is omitted as it is a trivial generalization of the soundness proof for the layered case given in Section 8. In particular, the order-preservation and adjointness conditions on multi-layered algebras take care of the rules (12s), (13s) and (14s).

Note that these sound interpretations apply to algebras that arise from multi-magmas as in Proposition 6.4; that is, in the case where $M=\mathcal{P}(\operatorname{Sg}(\mathcal{G}))$, for some ambient graph $\mathcal{G}$. Therefore, they apply to those that arise from the strong composition and bi-composition operators on graphs. In other words, formulae may be interpreted as sets of sub-graphs of $\mathcal{G}$ following the prescription above. 


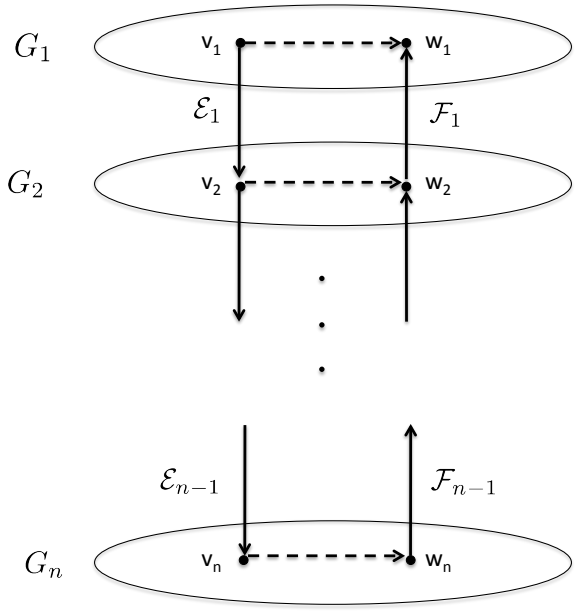

Figure 6. The Multi-layered Stack of Example 6.5

Example 6.5. Let $V(\mathcal{G})=\left\{v_{i} \mid 1 \leq i \leq n\right\} \cup\left\{w_{i} \mid 1 \leq i \leq n\right\}$. Let $e_{i}$ be an edge from $v_{i}$ to $v_{i+1}$, and $\mathcal{E}_{i}=\left\{e_{i}\right\}$ for all $1 \leq i<n$. Let $f_{i}$ be an edge from $w_{i+1}$ to $w_{i}$, and $\mathcal{F}_{i}=\left\{f_{i}\right\}$ for all $1 \leq i<n$. Let $c_{i}$ be an edge from $v_{i}$ to $w_{i}$, and $\mathcal{C}_{i}=\left\{c_{i}\right\}$ for all $1 \leq i \leq n$. Let $\mathcal{E}_{i}=\mathcal{F}_{i}=\emptyset$ for $i \geq n$. Let $\mathcal{C}_{i}=\emptyset$ for $i>n$. Let $E(\mathcal{G})=\bigcup_{i \geq 1} \mathcal{C}_{i} \cup \mathcal{E}_{i} \cup \mathcal{F}_{i}$.

Consider the interpretation on the layered algebra induced by strong bi-composition of subgraphs of $\mathcal{G}$ with respect to the sequences $\mathcal{E}_{1}, \ldots$ and $\mathcal{F}_{1}, \ldots$ For $1 \leq i \leq n$, let $\phi_{i}$ be valued so that $G \in \llbracket \phi_{i} \rrbracket=\mathcal{V}\left(\phi_{i}\right)$ iff $V(G)=\left\{v_{i}, w_{i}\right\}$ and there is no edge from $v_{i}$ to $w_{i}$ in $G$, for any sub-graph $G$. Let $\psi$ be valued so that $G \in \llbracket \psi \rrbracket=\mathcal{V}(\psi)$ iff there is no path in $G$ from $v_{1}$ to $w_{1}$. Then, suppressing all ambient graph and edge-set annotations, and presuming the evident definition of $\vDash$ for this variation of the language,

$$
G_{1} \vDash \rightarrow^{n-1, m}\left(\phi_{2}, \ldots, \phi_{n}, \psi\right)
$$

expresses the fact that if $G_{1}$ is stacked with layers $G_{2}, \ldots, G_{n}$, then no path from $v_{1}$ to $w_{1}$ is introduced. This is illustrated in Figure 6.

In the context of security examples, this can be used to specify the non-introduction of a back-channel upon the introduction of new layers to a system.

\section{Dynamics AND Examples}

The notion of layering that we have developed has many natural applications in complex systems modelling. One particularly appealing area of application lies in security, with others in a variety of network settings. We now formalize the three very simple, but illustrative, examples introduced in Section 1.

The presentation of these ideas depends on having a simple language of actions and an associated polymodal process or action logic, along the lines of Hennessy-Milner logic [27] or dynamic logic (e.g., [25]). Indeed, the bunched modal process logic introduced in [13, 11, 14, 12] would be a suitable candidate. Rather than develop the technical details of such a logic here, we just suppose we have action modalities $\langle a\rangle$ and $[a]$ whose meaning, informally for now, we describe below. The key requirement for our examples is that actions manipulate (i.e., consume, create, and move) resources, which are placed at locations.

We now extend LGL to a new logic LGLr in order to incorporate the above notion of resources at locations. This extension is based on an assignment of a of a set of resources $R$ to the vertices of the graph $G$. That is, each $r \in R$ is situated at a vertex of $G$. We denote such assignments 


$$
\begin{aligned}
& (\mathcal{G}, \mathcal{E}), G[R] \vDash_{\mathcal{E}} \top \quad \text { for all } G[R] \\
& (\mathcal{G}, \mathcal{E}), G[R] \vDash_{\mathcal{E}} \perp \quad \text { for no } \quad G[R] \\
& (\mathcal{G}, \mathcal{E}), G[R] \vDash_{\mathcal{E}} \mathrm{p} \quad \text { iff } \quad G[R] \in \mathcal{V}(\mathrm{p}) \\
& (\mathcal{G}, \mathcal{E}), G[R] \vDash_{\mathcal{E}} \phi \wedge \psi \quad \text { iff } \quad(\mathcal{G}, \mathcal{E}), G[R] \vDash_{\mathcal{E}} \phi \text { and }(\mathcal{G}, \mathcal{E}), G[R] \vDash_{\mathcal{E}} \psi \\
& (\mathcal{G}, \mathcal{E}), G[R] \vDash_{\mathcal{E}} \phi \vee \psi \quad \text { iff } \quad(\mathcal{G}, \mathcal{E}), G[R] \vDash_{\mathcal{E}} \phi \text { or }(\mathcal{G}, \mathcal{E}), G[R] \vDash_{\mathcal{E}} \psi \\
& (\mathcal{G}, \mathcal{E}), G[R] \vDash_{\mathcal{E}} \phi \rightarrow \psi \quad \text { iff } \quad(\mathcal{G}, \mathcal{E}), G[R] \vDash_{\mathcal{E}} \phi \text { implies }(\mathcal{G}, \mathcal{E}), G[R] \vDash_{\mathcal{E}} \psi \\
& (\mathcal{G}, \mathcal{E}), G[R] \vDash_{\mathcal{E}} \phi_{1} \triangleright \phi_{2} \quad \text { iff } \quad \text { for some } G_{1}, G_{2} \text { and } R_{1}, R_{2} \text { such that } \\
& G=G_{1} @_{\mathcal{E}} G_{2} \text { and } R=R_{1} \circ R_{2} \text {, } \\
& (\mathcal{G}, \mathcal{E}), G_{1}\left[R_{1}\right] \vDash_{\mathcal{E}} \phi_{1} \text { and }(\mathcal{G}, \mathcal{E}), G_{2}\left[R_{2}\right] \vDash_{\mathcal{E}} \phi_{2} \\
& (\mathcal{G}, \mathcal{E}), G[R] \vDash_{\mathcal{E}} \phi \rightarrow \psi \quad \text { iff } \quad \text { for all } H[S] \text { such that } G @_{\mathcal{E}} H \downarrow \text { and } R \circ S \downarrow \\
& (\mathcal{G}, \mathcal{E}), H[S] \vDash_{\mathcal{E}} \phi \text { implies }(\mathcal{G}, \mathcal{E}),(G @ \mathcal{E} H)[R \circ S] \vDash_{\mathcal{E}} \psi \\
& (\mathcal{G}, \mathcal{E}), G[R] \vDash_{\mathcal{E}} \phi \smile \psi \quad \text { iff } \quad \text { for all } H[S] \text { such that } H @_{\mathcal{E}} G \downarrow \text { and } R \circ S \downarrow \\
& (\mathcal{G}, \mathcal{E}), H[S] \vDash_{\mathcal{E}} \phi \text { implies }(\mathcal{G}, \mathcal{E}),\left(H @_{\mathcal{E}} G\right)[R \circ S] \vDash_{\mathcal{E}} \psi \\
& (\mathcal{G}, \mathcal{E}), G[R] \vDash_{\mathcal{E}}\langle a\rangle \phi \quad \text { iff } \quad \text { for some well-formed assignment } G\left[R^{\prime}\right] \\
& \text { such that } G[R] \stackrel{a}{\longrightarrow} G\left[R^{\prime}\right] \text {, } \\
& (\mathcal{G}, \mathcal{E}), G\left[R^{\prime}\right] \vDash_{\mathcal{E}} \phi \\
& (\mathcal{G}, \mathcal{E}), G[R] \vDash_{\mathcal{E}}[a] \phi \quad \text { iff } \quad \text { for all well-formed assignments } G\left[R^{\prime}\right] \\
& \text { such that } G[R] \stackrel{a}{\longrightarrow} G\left[R^{\prime}\right] \\
& (\mathcal{G}, \mathcal{E}), G\left[R^{\prime}\right] \vDash_{\mathcal{E}} \phi
\end{aligned}
$$

TABLE 4. The Satisfaction Relation for LGLr

as $G[R]$ where we think of $G$ as the (directed) graph of locations in a system model. Resources should also carry sufficient structure to allow some basic operations on resource elements. In $[35,41,40,13,11,14,12]$ resources are required to form pre-ordered partial monoids, such as the natural numbers $(\mathbb{N}, \leq,+, 0)$, and we use this approach here. Let $(\mathcal{R},=, \circ, e)$ be a resource monoid, where $\mathcal{R}$ is a collection of sets of resources and $\circ: \mathcal{R} \times \mathcal{R} \rightarrow \mathcal{R}$ is a commutative and associative binary operation, such as set union. Proposition 7.1, which is a minor adaptation of a result found in [12], illustrates that assignments of resources can be composed and that the algebraic semantics can be easily extended.

Proposition 7.1. Consider @ and ○. Both are binary operations with @ non-commutative and non-associative while $\circ$ is commutative and associative. A non-commutative, non-associative operation can be defined.

Proof. We have $@: \mathcal{G} \times \mathcal{G} \rightarrow \mathcal{G}$ and $\circ: \mathcal{R} \times \mathcal{R} \rightarrow \mathcal{R}$. Define $\bullet:(\mathcal{G} \times \mathcal{R}) \times(\mathcal{G} \times \mathcal{R}) \rightarrow(\mathcal{G} \times \mathcal{R})$ as $\left(G_{1}, R_{1}\right) \bullet\left(G_{2}, R_{2}\right)=\left(G_{1} @ G_{2}, R_{1} \circ R_{2}\right)$. It is clear that $\bullet$ is both non-commutative and non-associative.

The satisfaction relation for LGLr is shown in Table 4 (with the evident variations for bilayering). Using Proposition 7.1 and the notion of valuation from Section $4, \mathcal{V}$ now assigns atoms to sets of subgraphs of $\mathcal{G}$ that have assignments from $\mathcal{R}$.

Note that, in this simple set-up, actions simply manipulate the resources that are located at the vertices of the graph and that there is no manipulation of the structure of the graph $G$ itself. This 
formulation has no effect on the semantics of the other (propositional) connectives that we have previously established. This simple set-up is sufficient to handle the examples that we consider in this section, but richer classes of actions might also be considered. We abstract away from resources in Examples 7.1 and 7.2 and therefore omit the resource assignments as they are not required. The notion is, however, crucial in presenting Example 7.3.

7.1. Example: Email Clients and Servers. Consider the sending of an email message from one client to another, as depicted in Figure 7. From the users' perspective, the message is sent (via the action send, in the Client Layer) from Client 1 to Client 2. In fact, the message is sent over the internet (in the IP Layer) from an outgoing server (Server 1) to an incoming server (Server 2) as a collection of packets.

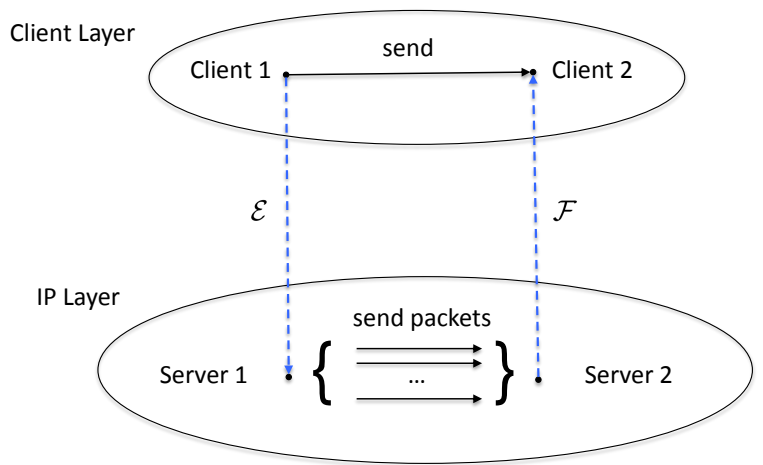

Figure 7. Email Clients and Servers in the IP Stack

Associated with Clients 1 and 2 and Servers 1 and 2, respectively, are correctness conditions $\phi_{1}$ and $\phi_{2}$ and $\psi_{1}$ and $\psi_{2}$. In the Client Layer, if $\phi_{1}$ holds, then Client 1 is able to send an email message to Client 2. Assuming $\phi_{2}$ holds, Client 2 displays the message. From the users' perspectives, interacting with the clients, this is all that is observed. What actually happens - at our present level of abstraction, at least, simplifying to a single action the process of communication with the server and splitting the message into packets - is, given that $\phi_{1}$ holds, an action split sends the message to Server 1 along an edge in the edge set $\mathcal{E}$, in the IP Layer. More generally, the full IP stack can be characterized in our terms using the notion of stack as defined in Section 5 .

Then, provided $\psi_{1}$ holds, Server 1 sends the constituent packets of the message over the internet to Server 2. Call this action, which interleaves the packets with other traffic, interleave (this is synonymous with the send packets action in Figure 7). An action combine (simplifying as above) then sends the reassembled email message to Client 2 along an edge in $\mathcal{F}$.

Thus, let $G_{1}$ be the graph of the Client Layer and let $G_{2}$ be the graph of the IP Layer, so that $\mathcal{G}=G=G_{1} \hat{\Theta}_{\mathcal{E}, \mathcal{F}} G_{2}$. If $\chi_{1}$ and $\chi_{2}$ describe the remaining properties, or state, of the Client and IP Layers, respectively, then we have

$$
(\mathcal{G},\langle\mathcal{E}, \mathcal{F}\rangle), G_{1} \quad \vDash_{\mathcal{E}, \mathcal{F}} \quad \theta_{1},
$$

where $\theta_{1}=\left(\phi_{1} \wedge\langle\right.$ send $\left.\rangle \phi_{2}\right) \wedge \chi_{1}$, and

$$
(\mathcal{G},\langle\mathcal{E}, \mathcal{F}\rangle), G_{2} \quad \vDash_{\mathcal{E}, \mathcal{F}} \quad \theta_{2},
$$

where $\theta_{2}=\left(\psi_{1} \wedge\langle\right.$ interleave $\left.\rangle \psi_{2}\right) \wedge \chi_{2}$. The $\phi$ s and $\psi$ s describe the (somewhat simplified) specific properties of the transmission of the message and the $\chi_{\mathrm{s}}$ describe the remaining properties (or states) of the layers. In these instances of the satisfaction relation, $\vDash_{\mathcal{E}, \mathcal{F}}$, we have, for simplicity, suppressed mention of components of the state other than the (location) graph component, although formally other components are required to support the $\phi$ s and $\psi \mathrm{s}$. A transition system on states is also formally required to support the modal operators: this could either be done in an ad hoc way, following the textual specifications above, or better in a process calculus. Importantly, note that none of the actions considered here modifies the graph in any way. These conventions apply to all subsequent uses of satisfaction relations. 
Now consider the transmission of the message - more precisely, the resources that constitute the message - between layers. Here the use of scaffolds seems essential. To see this, we consider some properties of split and combine that must hold. For example, we need the semantics of split, which moves resources between the upper and lower layers, to reflect structure of layers and to establish the necessary correctness condition. Similarly for combine. Thus we have

$$
(\mathcal{G}, \mathcal{E}), G \quad \vDash_{\mathcal{E}} \quad\langle\text { split }\rangle \psi_{1} \wedge\left(\theta_{1} \triangleright \theta_{2}\right)
$$

and

$$
(\mathcal{G}, \mathcal{F}), G \quad \vDash_{\mathcal{F}} \quad\langle\text { combine }\rangle \phi_{2} \wedge\left(\theta_{2} \triangleright \theta_{1}\right) .
$$

Notice here that in the judgement 5 we have the subformula $\theta_{1}-\theta_{2}$ and that in the judgement 6 we have the subformula $\theta_{2}-\theta_{1}$, reflecting the fact that the former refers to the layering, with respect to the edge set $\mathcal{E}$, of $G_{1}$ over $G_{2}$ and the latter refers to the layering, with respect to the edge set $\mathcal{F}$, of $G_{2}$ over $G_{1}$. We make use here not only of the multiplicative connectives that are directly associated with the structure of the individual layers and their combination, but also of the (usual) additives in order to describe properties of the whole graph including the inter-layer edges. In the judgements 5 and 6 , we have properties of the whole graph, the former picking out the transmission of the message from the sending client to outgoing server (via the layering with respect to $\mathcal{E}$ ) and the latter picking out the transmission from incoming server to the receiving client (via the layering with respect to $\mathcal{F}$ ). We can then write

$$
(\mathcal{G},\langle\mathcal{E}, \mathcal{F}\rangle), G \quad \vDash_{\mathcal{E}, \mathcal{F}} \quad\langle\text { split }\rangle \psi_{1} \wedge\langle\text { combine }\rangle \phi_{2} \wedge\left(\theta_{1} \triangleright \theta_{2}\right) .
$$

Rather than using a family of satisfaction relations as above, an alternative logical language for this example could use a single satisfaction relation and families of multiplicative connectives that are explicitly indexed by their defining distinguished edge sets, for example, $\mathcal{E}$ and $\mathcal{F}$. However, this alternative would not generalize easily to allow for examples in which the ambient graph $\mathcal{G}$ is modified by actions.

7.2. Example: Avoiding the Security Barrier. Consider the example illustrated in Figure 8, which corresponds to a well-known photograph of such a situation (see, for example, Schneier's blog [45]). Here we see a security barrier that controls access along a road that connects two other roads (the outside and the inside, say). The problem, of course, is that in the absence of a fence, the barrier does not effectively control access to the inside because it is possible for vehicles to swerve around it.

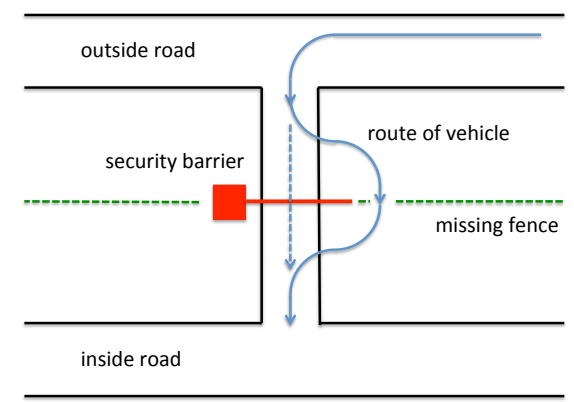

Figure 8. Pictorial Representation of the Security Barrier Photograph [45]

This situation, which is much simpler than the IP example, can also be described quite neatly in our logic of layered graphs. Consider the graph, $\mathcal{G}$, of locations that underlies this model. The graph can be seen as having two layers, the Routes Layer, giving the paths that a vehicle can take, and the Security Architecture Layer. In this example, depicted in Figure 9, the edges between the two layers merely identify the out and in locations in each layer. Associated with being on the inside is a property $\phi_{\text {token }}$ of possessing the access control token (such as an identity card) that 


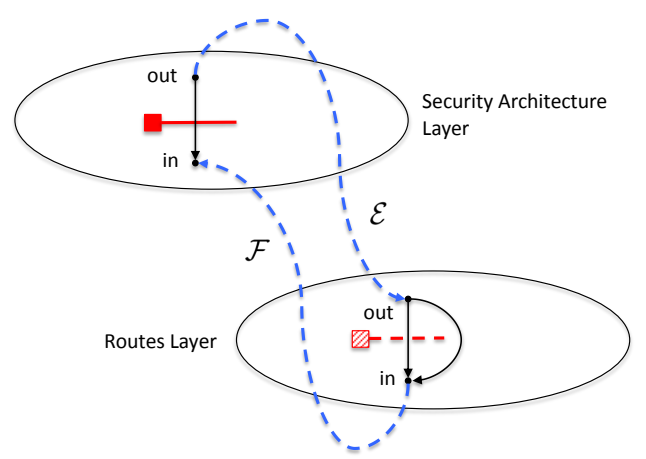

Figure 9. Security Barrier Locations Graph

is required to pass the barrier. Thus, in the Security Architecture Layer, with graph $G_{1}$, it is the case that a vehicle can pass inside, then it will possess the necessary access control token:

$$
(\mathcal{G},\langle\mathcal{E}, \mathcal{F}\rangle), G_{1} \quad \vDash_{\mathcal{E}, \mathcal{F}} \quad\langle\text { pass }\rangle \phi_{\text {in }} \rightarrow \phi_{\text {token }} .
$$

In the Routes Layer, however, it is possible to swerve past the barrier, and thereby be inside, without being in possession of the token:

$$
(\mathcal{G},\langle\mathcal{E}, \mathcal{F}\rangle), G_{2} \quad \vDash_{\mathcal{E}, \mathcal{F}} \quad\langle\text { swerve }\rangle \phi_{\text {in }} \rightarrow \neg \phi_{\text {token }} .
$$

Thus the existence of the weak link is characterized by the formula

$$
(\mathcal{G},\langle\mathcal{E}, \mathcal{F}\rangle), G_{1} \hat{\bigotimes}_{\mathcal{E}, \mathcal{F}} G_{2} \quad \vDash_{\mathcal{E}, \mathcal{F}} \quad\left(\langle\text { pass }\rangle \phi_{\text {in }} \rightarrow \phi_{\text {token }}\right) \triangleright\left(\langle\text { swerve }\rangle \phi_{\text {in }} \rightarrow \neg \phi_{\text {token }}\right),
$$

which has a component in which both the inside can be accessed and the access control is undermined.

Note that in this example the layers represent only distinct conceptual aspects of a real system, with inter-layer links representing the shared substrate of the system. In contrast, in the IP example, a system is constructed that realizes both the conceptual layers as separate entities, and also the connections between them.

7.3. Example: Data Security Breach via Mobile Devices. Let $R$ denote some set of data resources assigned to the vertices of a graph as described above. Let $r$ denote an element of $R$, and let $\phi$ be a property such that $\phi(r)$ holds at the vertex of $G$, where $r$ is assigned by $G[R]$.

Consider an organization whose information network is partitioned into General and Secure sub-networks. The organization's intention is that no data be permitted to pass between the General and Secure Networks. In the presence of mobile storage devices (such as a USB memory sticks), this intended property can be difficult to maintain. In this example, we use our logical account of layering to give a characterization of this situation, which can be captured using a graph made up of two layers, a Network Layer and a Mobile Storage Layer. This example illustrates the use of the implication

Associated with the system is the resource $r$ that represents secure data within the Network Layer. Let $G_{1}$ denote the Network Layer, $G_{G}$ denote the General Network and $G_{S}$ denote the Secure Network so $G_{1}=G_{G} \cup G_{S}$. Let $G_{2}$ denote the Mobile Storage Layer and suppose $\mathcal{G}$ is such that $\mathcal{G}=G_{1} \hat{@}_{\mathcal{E}, \mathcal{F}} G_{2}$. Associated with the Mobile Storage Layer are actions copy, download, and upload. The action copy takes data $r$ from one location in $G_{2}$ and copies it to another location (assuming the data is held on some sort of mobile storage device), while download and upload copy data along edges of $\mathcal{E}$ and $\mathcal{F}$ respectively.

For any resource, the Network Layer uses local compliance properties. The property $\chi_{S}$ describes compliance with a policy allowing the resource to be in $G_{S}$ and $\chi_{G}$ asserts that if a resource is not permitted in $G_{G}$, then it is not in $G_{G}$. That is, secure data is never present at any node of $G_{G}$.

In the Mobile Storage Layer, there are Locations (vertices) 1 and 2 that allow individuals to download and upload data from and to the Network Layer. The copy action occurs along links 


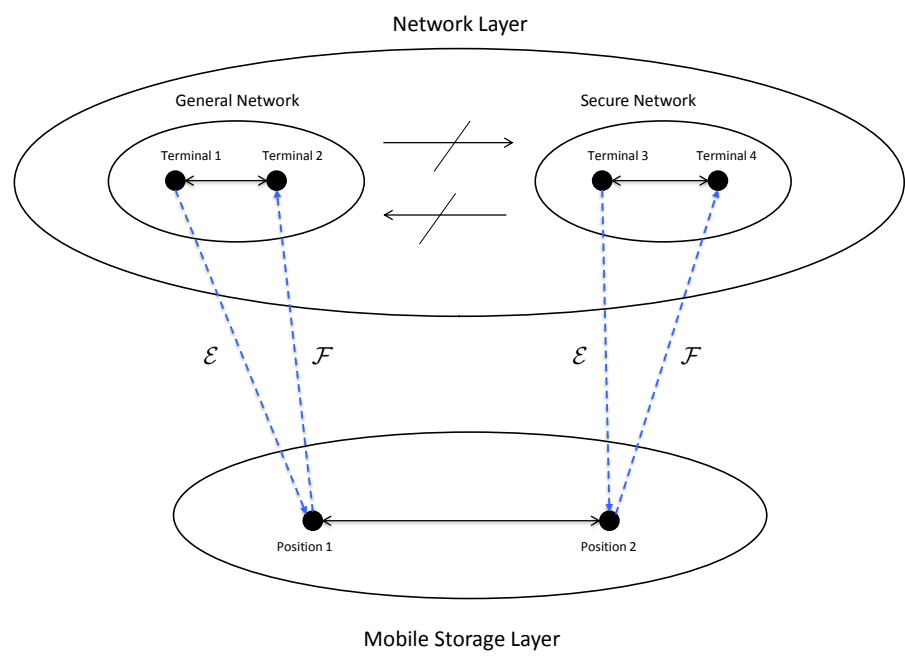

FiguRE 10. Data Segregation and Mobile Storage Graph

within the Mobile Storage Layer. A security breach arises when an individual with sufficient security privileges downloads data from the Secure Network to Location 2, copies (e.g., using a USB memory stick) the data to Location 1 , and then uploads it to the General Network. Let $R$ represent the set of data resources associated to a vertex in the Secure Network and let $R_{2}$ be $R$ extended with a copy of $r$ at Location 2 , downloaded from $G_{S}$.

The Mobile Storage Layer also uses a local compliance property: $\theta$ describes compliance with a policy allowing a resource to be in $G_{2}$. Therefore, in order to copy successfully secure data $r$ within $G_{2}$, we must have that

$$
(\mathcal{G},\langle\mathcal{E}, \mathcal{F}\rangle), G_{2}\left[R_{2}\right] \quad \vDash_{\mathcal{E}, \mathcal{F}} \quad\langle\text { copy }\rangle \theta(r) .
$$

The failure of data segregation is highlighted when considering how the required security properties can be satisfied by the layered structure. Suppose that

$$
(\mathcal{G},\langle\mathcal{E}, \mathcal{F}\rangle),\left(G_{1} @_{\mathcal{E}, \mathcal{F}} G_{2}\right)[R] \quad \vDash_{\mathcal{E}, \mathcal{F}} \quad\langle\text { download }\rangle\left(\chi_{S}(r) \triangleright \theta(r)\right)
$$

and

$$
(\mathcal{G},\langle\mathcal{E}, \mathcal{F}\rangle),\left(G_{1} @_{\mathcal{E}, \mathcal{F}} G_{2}\right)\left[R_{2}\right] \quad \vDash_{\mathcal{E}, \mathcal{F}} \quad\langle\text { copy }\rangle\langle\operatorname{upload}\rangle\left(\left(\neg \chi_{G}(r)\right) \triangleright \theta(r)\right) .
$$

Property 11 represents the situation in which secure data is copied onto a mobile storage device within the Mobile Storage Layer. This situation satisfies all required properties of the Mobile Storage Layer, along with the condition $\chi_{S}(r)$, which describes compliance with the policy allowing $r$ to be in $G_{S}$. Property 12 says that secure data might be copied within the Mobile Storage Layer before being uploaded into the General Network, which would no longer comply with $\chi_{G}$. In this situation, the ability to copy $r$ in $\mathcal{F}$ and upload it to $G_{G}$ will lead to an assignment of $r$ within $G_{G}$ when it should be the case that $(\mathcal{G},\langle\mathcal{E}, \mathcal{F}\rangle), G_{1}\left[R^{\prime}\right] \not \models \chi_{G}(r)$, where $R^{\prime}$ is the assignment of resources in $G_{G}$ that results from the copy and upload.

The following relation describes how a failure of compliance with $\chi_{G}$ arises from the ability to copy and upload a resource that has been downloaded from $G_{S}$ :

$$
\begin{aligned}
(\mathcal{G},\langle\mathcal{E}, \mathcal{F}\rangle),\left(G_{1} @_{\mathcal{E}, \mathcal{F}} G_{2}\right)\left[R_{2}\right] \vDash_{\mathcal{E}, \mathcal{F}} & \langle\text { download }\rangle\left(\chi_{S}(r) \triangleright \theta(r)\right) \wedge \\
& \langle\text { copy }\rangle\langle\operatorname{upload}\rangle\left(\left(\neg \chi_{G}(r)\right) \triangleright \theta(r)\right) .
\end{aligned}
$$

Note that we assume here that (11) continues to hold for assignment $R_{2}$ at $G_{2}$ (recall that $R_{2}$ simply adds a copy of $r$ at Location 2). 
Now, note that $(\mathcal{G},\langle\mathcal{E}, \mathcal{F}\rangle), G_{1}[R] \vDash_{\mathcal{E}, \mathcal{F}} \chi_{G}(r)$. However,

$$
(\mathcal{G},\langle\mathcal{E}, \mathcal{F}\rangle), G_{1}\left[R_{2}\right] \quad \vDash_{\mathcal{E}, \mathcal{F}} \quad \theta(r)
$$

$$
\left(\langle\text { download }\rangle\left(\chi_{S}(r) \triangleright \theta(r)\right) \wedge\langle\text { copy }\rangle\langle\text { upload }\rangle\left(\left(\neg \chi_{G}(r)\right) \triangleright \theta(r)\right)\right)
$$

demonstrating that the security policy $\chi_{G}$ can be violated as result of the introduction of a new layer; in particular, as witnessed in (13), which is obtained by unpacking the satisfaction of the $\rightarrow$ formula in (14), using $(\mathcal{G},\langle\mathcal{E}, \mathcal{F}\rangle), G_{2}\left[R_{2}\right] \vDash_{\mathcal{E}, \mathcal{F}} \theta(r)$.

This example makes use of the $\rightarrow$ implication. Had we instead considered the Mobile Storage Layer to be layered over the Network Layer, then the example would have been constructed using the operator, with all other details being analogous.

\section{Soundness and Completeness}

Consider the notion of interpretation of formulae of LGL on an arbitrary layered algebra $\mathbb{A}$ as set out in Section 4. We now show that the rules of the calculus $\mathbf{L G L}_{\mathrm{H}}$ set out in Section 3 are sound with respect to this interpretation. The soundness of the interpretations on layered magmas and scaffolds are immediate corollaries, given the results of Propositions 4.1 and 4.2.

Theorem 8.1. The rules of $\mathbf{L G L}_{\mathrm{H}}$ are sound on layered algebras: for any layered algebra $\mathbb{A}$, for any interpretation $\llbracket-\rrbracket:$ Formulae $\longrightarrow \mathbb{A}$, and for any propositions $\phi$ and $\psi$, if $\phi \vdash \psi$ then $\llbracket \phi \rrbracket \leq \llbracket \psi \rrbracket$.

Proof. The proof of this result is a standard induction on the structure of derivations, with the key points being: that $\mathbb{A}$ is a Boolean algebra, which takes care of axioms 1-4 and rules 5-11 of Table 1 ; that $\longrightarrow$ on $\mathbb{A}$ is a bifunctor, which handles rule 12 ; that $\rightarrow$ and - on $\mathbb{A}$ are respective adjoints to the (single-argument) functors induced by $\boldsymbol{\bullet}$, which deals with rules 13-16.

We now consider completeness of the given calculus with respect to layered algebras.

Proposition 8.2. The following properties hold for all propositions $\phi, \psi, \eta$ :

(1) $\eta \vdash \phi \wedge \psi$ if and only if $\eta \vdash \phi$ and $\eta \vdash \psi$;

(2) $\eta \vdash \phi \rightarrow \psi$ if and only if, for all $\xi$, if $\xi \vdash \phi$ then $\eta \triangleright \xi \vdash \psi$;

(3) $\eta \vdash \phi-\psi$ if and only if, for all $\xi$, if $\xi \vdash \phi$ then $\xi \triangleright \eta \vdash \psi$.

The proof of each is by immediate application of the axioms and rules, and is omitted.

Lemma 8.3. There is a layered algebra $\mathcal{T}$ and an interpretation $\llbracket-\rrbracket_{\mathcal{T}}:$ Formulae $\longrightarrow \mathbb{A}$ such that, for all propositions $\phi$ and $\psi$, if $\phi \nvdash \psi$ then $\llbracket \phi \rrbracket_{\mathcal{T}} \not \leq \llbracket \psi \rrbracket_{\mathcal{T}}$.

Proof. We construct a layered algebra $\mathcal{T}=(\mathcal{T}, \wedge, \neg, \top, \neg, \rightarrow, \neg)$ as follows:

- Let $\mathcal{T}$ be a set of equivalence classes of propositions with respect to the relation of provability; elements of $\mathcal{T}$ are written $\lceil\phi\rceil,\lceil\psi\rceil$ etc.; note that $\lceil\phi\rceil=\lceil\psi\rceil$ if and only if $\phi \vdash \psi$ and $\psi \vdash \phi$, for any $\phi$ and $\psi$; transitivity of the equivalence comes from Proposition 3.1;

- Define a binary relation $\leq$ on $\mathcal{T}$ by $\lceil\phi\rceil \leq\lceil\psi\rceil$ iff $\phi \vdash \psi$;

- Define the element $T=\lceil\top\rceil$;

- Define the complementation operation by $\neg\lceil\phi\rceil=\lceil\phi \rightarrow \perp\rceil$;

- Define the conjunction operation by $\lceil\phi\rceil \wedge\lceil\psi\rceil=\lceil\phi \wedge \psi\rceil$;

- Define the monoidal composition by $\lceil\phi\rceil \neg\lceil\psi\rceil=\lceil\phi \downarrow \psi\rceil ;$

- Define the operation $\rightarrow$ by $\lceil\phi\rceil \rightarrow\lceil\psi\rceil=\lceil\phi \rightarrow \psi\rceil ;$

- Define the operation $\neg$ by $\lceil\phi\rceil\llcorner\lceil\psi\rceil=\lceil\phi \smile \psi\rceil$.

The order $\leq$ is a partial order, with transitivity coming from Proposition 3.1. The remaining checks that the operations are well-defined and that this structure is indeed a layered algebra are of a standard kind. In particular, they use the axioms and rules of Table 1, the properties of Proposition 8.2 and Proposition 3.1.

Define a valuation $\mathcal{V}$ : Atoms $\longrightarrow \mathcal{T}=\lceil\phi\rceil$ by $\mathcal{V}(\phi)=\lceil\phi\rceil$. Using the operations above, this valuation extends to an interpretation that satisfies the property $\llbracket \phi \rrbracket_{\mathcal{T}}=\lceil\phi\rceil$ on all formulae $\phi$. If $\phi \nvdash \psi$ then $\lceil\phi\rceil \not \subset\lceil\psi\rceil$ by construction, and so $\llbracket \phi \rrbracket_{\mathcal{T}} \not \leq \llbracket \psi \rrbracket_{\mathcal{T}}$. 
The completeness theorem (for layered algebras) is an immediate corollary to the above term model construction.

Theorem 8.4. For any propositions $\phi$ and $\psi$ of $\mathbf{L G L}$, if $\llbracket \phi \rrbracket \leq \llbracket \psi \rrbracket$ for all interpretations $\llbracket-\rrbracket$ in all layered algebras, then $\phi \vdash \psi$ in $\mathbf{L G L}_{\mathrm{H}}$.

Further discussions of the use of algebraic structures for (commutative) bunched logic can be found in $[41,20]$. The issue of completeness for boolean variants of BI with respect to monoidal models is complicated, and we do not study completeness for $\mathbf{L} \mathbf{G} \mathbf{L}_{\mathrm{H}}$ with respect to layered magmas here.

\section{Some Proof Theory}

Proofs in BI can be presented in a variety of formal calculi [35, 40, 41, 7] — as a Hilbert system, as a sequent calculus [21], as a natural deduction system [38], and as a display calculus [3].

We now examine various calculi for proofs in LGL. We have already presented a Hilbert system $\mathbf{L} \mathbf{G L}_{\mathrm{H}}$. In this section, we first give a natural deduction calculus, $\mathbf{L} \mathbf{G} \mathbf{L}_{\mathrm{ND}}$, and then give a sequent calculus, $\mathbf{L G L}_{\mathrm{SC}}$. These presentations follow those of similar systems by, for example, Lambek [30, 31, 32, 33], Girard [22] and Pym [39, 40]. We also give a display calculus, $\mathbf{L G L}_{\mathrm{DC}}$, following techniques laid out by Brotherston and Calcagno [7, 8]. This calculus, which has notably better meta-theoretical properties than either the natural deduction or sequent calculi, allows an appropriate cut-elimination result to be obtained.

The calculi $\mathbf{L} \mathbf{G L}_{\mathrm{ND}}$ and $\mathbf{L} \mathbf{G} \mathbf{L}_{\mathrm{SC}}$ both allow for the derivation of judgements of the form $\Gamma \vdash \phi$, where $\Gamma$ is a context (a structured collection of propositions) and $\phi$ is a single proposition. For both $\mathbf{L G L}_{\mathrm{ND}}$ and $\mathbf{L} \mathbf{G L}_{\mathrm{SC}}$ the appropriate notion of context for logical judgements is that of bunch, as described in [35]. We reserve $\Gamma, \Delta, \Theta$ for bunches. Bunches are generated by the grammar

$$
\Gamma:=\emptyset|\phi| \Gamma ; \Gamma \mid \Gamma, \Gamma
$$

where $\emptyset$ is the empty context and $\phi$ is any proposition. The symbol ';' is the additive context former, and the symbol ',' is the multiplicative context former.

We use the notation $\Gamma(\Delta)$ to indicate a bunch with a distinguished sub-bunch $\Delta$, that is, a particular sub-tree. We write $\Gamma\left[\Delta^{\prime} / \Delta\right]$ or $\Gamma\left(\Delta^{\prime}\right)$ for the bunch formed by substituting a bunch $\Delta^{\prime}$ for the sub-bunch $\Delta$ in $\Gamma$. Brackets are also used to disambiguate the tree structure of bunches. The context-formers have structural properties that match those of the logical conjunctions to which they correspond. There should be exchange, associativity, weakening and contraction rules for the additive context former. The multiplicative context former is not required to have any of these properties. This is in contrast to the situation in bunched logic where it is commutative and associative [35]. In order to capture the structural properties, we define an equivalence relation $\equiv$ on bunches. This is done by first of all imposing unit, commutativity and associativity relations as follows: $\emptyset ; \Gamma \equiv \Gamma, \Gamma ; \Delta \equiv \Delta ; \Gamma$, and $\Gamma ;(\Delta ; \Theta) \equiv(\Gamma ; \Delta) ; \Theta$. We then close this off to a congruence relation in the standard way: if $\Gamma \equiv \Delta$ and $\Delta \equiv \Theta$, then $\Gamma \equiv \Theta$ holds; if $\Delta \equiv \Delta^{\prime}$, then $\Gamma\left(\Delta^{\prime}\right) \equiv \Gamma(\Delta)$. Our formulation here does not use a multiplicative unit bunch.

The natural deduction calculus $\mathbf{L} \mathbf{G L}_{\mathrm{ND}}$ is specified by the rules given in Table 5 . The sequent calculus $\mathbf{L} \mathbf{G} \mathbf{L}_{\mathrm{SC}}$ is specified by the rules given in Table 6 .

We now introduce the display calculus $\mathbf{L G L}_{\mathrm{DC}}$. First, we make a small change to the language, so that $\neg \phi$ is no longer identified with $\phi \rightarrow \perp$. However, any such pair of propositions will turn out to be logically equivalent. Display calculi use structured collections of propositions called structures that generalize bunches. We use the letters $\Gamma, \Delta, \Theta$ for structures. Structures are constructed using additive and multiplicative structural connectives. The grammar of structures is as follows:

$$
\Gamma:=\emptyset|\phi| \# \Gamma|\Gamma ; \Gamma| \Gamma, \Gamma|\Gamma \bullet \Gamma| \Gamma \bullet \Gamma .
$$

A consecution is an ordered pair of structures, written in the form $\Gamma \vdash \Delta$. We say that $\Gamma$ is the antecedent and $\Delta$ is the consequent.

Table 7 indicates the division of structure formers into additives and multiplicatives, and their intended logical meaning - this depends upon whether they appear in an antecedent or consequent 


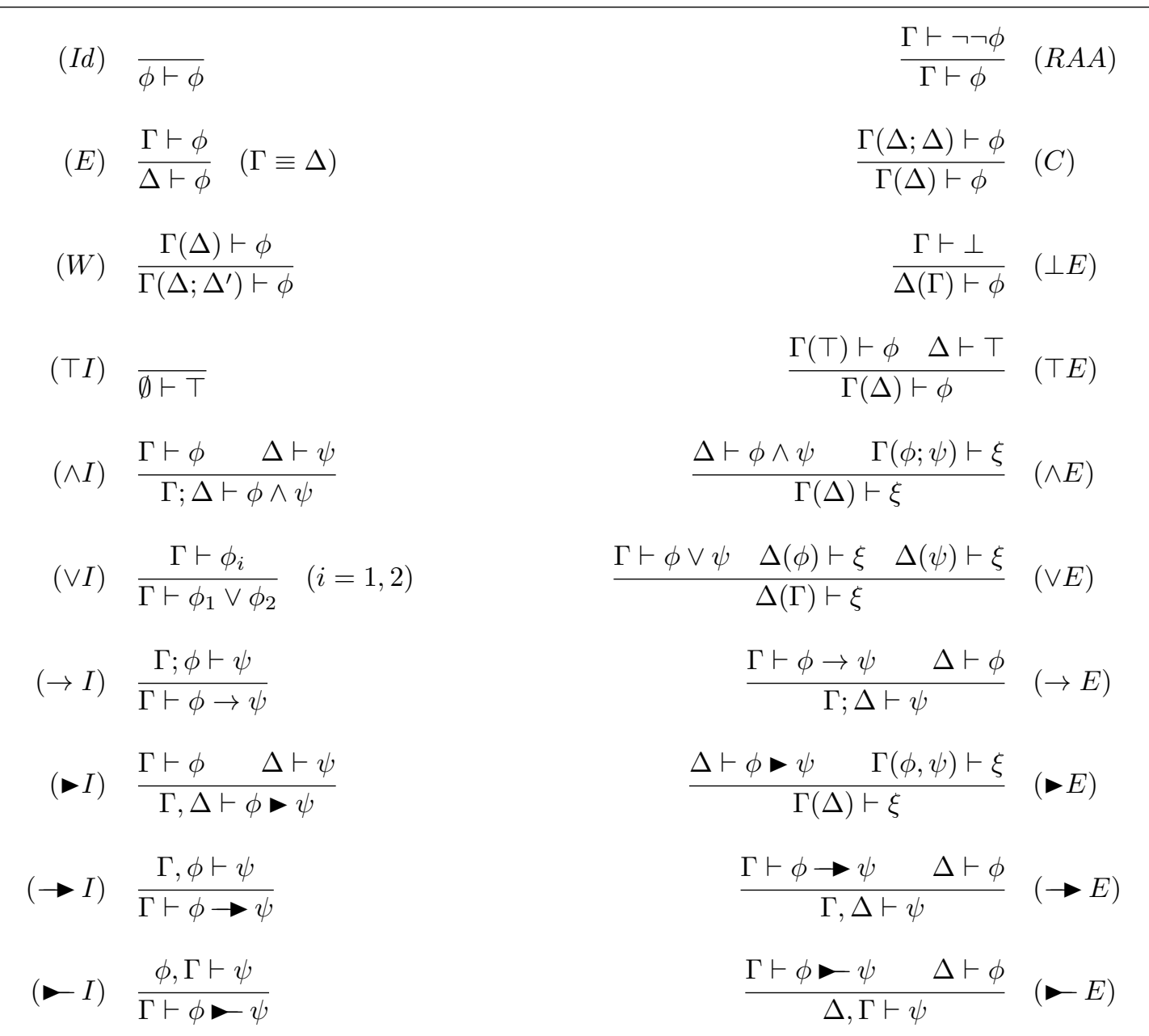

TABLE 5. Natural Deduction System LGL $\mathbf{L}_{\mathrm{ND}}$

part of a consecution (as explained below). Note that there is no multiplicative unit (truth) or negation, but that there are two multiplicative implications.

A substructure or part of a structure $\Gamma$ is a subtree of the syntax tree for $\Gamma$ such that all leaves are propositions. For any structure $\Gamma$, each substructure is classified as either a positive part or a negative part, according to the following recursive definition:

- $\Gamma$ is a positive part of $\Gamma$,

- if $\Delta$ is a negative (respectively, positive) part of $\Gamma$, then it is a positive (respectively, negative) part of $\# \Gamma$,

- if $\Delta$ is a negative (positive) part of exactly one of $\Gamma_{1}$ or $\Gamma_{2}$, then it is a negative (positive) part of $\Gamma_{1} ; \Gamma_{2}$ and $\Gamma_{1}, \Gamma_{2}$,

- if $\Delta$ is a part of exactly one of $\Gamma_{1}$ or $\Gamma_{2}$, and if $\Delta$ is a negative (positive) part of $\Gamma_{1}$ or a positive (negative) part of $\Gamma_{2}$, then it is a positive (negative) part of $\Gamma_{1} \bullet-\Gamma_{2}$ and $\Gamma_{1} \bullet \Gamma_{2}$.

A substructure $\Theta$ occurring in exactly one of $\Gamma$ or $\Delta$ is said to be an antecedent (respectively, consequent) part of a consecution $\Gamma \vdash \Delta$ if it a positive (respectively, negative) part of $\Gamma$ or a negative (respectively, positive) part of $\Delta$.

Where the meaning of a structure connective is undefined (according to Table 7) in a part, that connective is not allowed to occur as the principal connective in such a part of any consecution. 


$$
\begin{aligned}
& \text { (Id) } \overline{\phi \vdash \phi} \\
& \frac{\Gamma \vdash \neg \neg \phi}{\Gamma \vdash \phi} \quad(R A A) \\
& \text { (E) } \frac{\Gamma \vdash \phi}{\Delta \vdash \phi} \quad(\Gamma \equiv \Delta) \\
& \frac{\Gamma(\Delta ; \Delta) \vdash \phi}{\Gamma(\Delta) \vdash \phi} \\
& (W) \frac{\Gamma(\Delta) \vdash \phi}{\Gamma\left(\Delta ; \Delta^{\prime}\right) \vdash \phi} \\
& \frac{\Gamma \vdash \phi \quad \Delta(\phi) \vdash \xi}{\Delta(\Gamma) \vdash \xi} \\
& (\perp L) \quad \overline{\Gamma(\perp) \vdash \phi} \\
& (\top L) \frac{\Gamma(\emptyset) \vdash \phi}{\Gamma(\top) \vdash \phi} \\
& \overline{\emptyset \vdash \top} \\
& (\wedge L) \frac{\Gamma(\phi ; \psi) \vdash \xi}{\Gamma(\phi \wedge \psi) \vdash \xi} \\
& \frac{\Gamma \vdash \phi \quad \Delta \vdash \psi}{\Gamma ; \Delta \vdash \phi \wedge \psi} \\
& (\vee L) \frac{\Gamma(\phi) \vdash \xi \quad \Gamma(\psi) \vdash \xi}{\Gamma(\psi \vee \psi) \vdash \xi} \\
& (i=1,2) \frac{\Gamma \vdash \phi_{i}}{\Gamma \vdash \phi_{1} \vee \phi_{2}} \quad(\vee R) \\
& (\rightarrow L) \frac{\Delta \vdash \phi \quad \Gamma(\psi) \vdash \xi}{\Gamma(\Delta ; \phi \rightarrow \psi) \vdash \xi} \\
& \frac{\Gamma ; \phi \vdash \psi}{\Gamma \vdash \phi \rightarrow \psi} \quad(\rightarrow R) \\
& \left(\llcorner L) \frac{\Gamma(\phi, \psi) \vdash \xi}{\Gamma(\phi \triangleright \psi) \vdash \xi}\right. \\
& \frac{\Gamma \vdash \phi \quad \Delta \vdash \psi}{\Gamma, \Delta \vdash \phi \triangleright \psi} \quad(R) \\
& (\rightarrow L) \frac{\Gamma \vdash \phi \quad \Delta(\psi) \vdash \xi}{\Delta(\phi \rightarrow \psi, \Gamma) \vdash \xi} \\
& \frac{\Gamma, \phi \vdash \psi}{\Gamma \vdash \phi \rightarrow \psi} \quad(\rightarrow R) \\
& \text { L) } \frac{\Gamma \vdash \phi \quad \Delta(\psi) \vdash \xi}{\Delta(\Gamma, \phi \longleftarrow \psi) \vdash \xi} \\
& \frac{\phi, \Gamma \vdash \psi}{\Gamma \vdash \phi} \psi(\llcorner R)
\end{aligned}
$$

TABLE 6. Sequent Calculus $\mathbf{L G L}_{\mathrm{SC}}$

\begin{tabular}{cccc} 
& & & \\
Connective & Additive/Multiplicative & Antecedent & Consequent \\
\hline$\emptyset$ & A & truth & falsity \\
$\#$ & A & negation & negation \\
$;$ & A & conjunction & disjunction \\
, & M & conjunction & undefined \\
$\rightarrow$ & M & undefined & implication (right, $\rightarrow$ ) \\
$\bullet$ & M & undefined & implication (left,
\end{tabular}

TABLE 7. Diplay Calculus Structure Connectives

We call this the structure-position condition. This restriction is enforced by the derivation system for consecutions set out below.

Let \langle\rangle$_{D}$ be a binary relation on consecutions. Let $\equiv_{D}$ be the equivalence relation given by the reflexive, symmetric, transitive closure of \langle\rangle$_{D}$. The relation $\equiv_{D}$ is called a display 
equivalence if: for any antecedent part $\Theta$ of $\Gamma \vdash \Delta$, it is possible to construct a structure $\Lambda$ such that $\Gamma \vdash \Delta \equiv_{D} \Theta \vdash \Lambda$, and for any consequent part $\Theta$ of $\Gamma \vdash \Delta$, it is possible to construct a structure $\Lambda$ such that $\Gamma \vdash \Delta \equiv_{D} \Lambda \vdash \Theta$.

The binary relation \langle\rangle$_{D}$ for the present calculus is defined by a set of axioms known as display postulates. Instances of the relation defined by the display postulates take the form $C\langle\rangle_{D} C^{\prime}$, where $C$ and $C^{\prime}$ are consecutions. The display postulates are shown in Table 8 .

$$
\begin{array}{lll}
\Gamma ; \Delta \vdash \Theta\langle\rangle_{D} \Gamma \vdash \# \Delta ; \Theta\langle\rangle_{D} \Delta ; \Gamma \vdash \Theta & \Gamma, \Delta \vdash \Theta\langle\rangle_{D} \Gamma \vdash \Delta \rightarrow \Theta \\
\Gamma \vdash \Delta ; \Theta\langle\rangle_{D} \Gamma ; \# \Delta \vdash \Theta\langle\rangle_{D} \Gamma \vdash \Theta ; \Delta & \Delta, \Gamma \vdash \Theta\langle\rangle_{D} \Gamma \vdash \Delta \bullet \Theta \\
\Gamma \vdash \Delta\langle\rangle_{D} \# \Delta \vdash \# \Gamma\langle\rangle_{D} \# \# \Gamma \vdash \Delta &
\end{array}
$$

TABLE 8. Display Postulates for $\mathbf{L G L}_{\mathrm{DC}}$

The display calculus, $\mathbf{L} \mathbf{G L}_{\mathrm{DC}}$, is given by the following: the set of formulae and formulae connectives of LGL; the set of structures and structure connectives as defined above; the set of display postulates (given in Table 8) that generate the relation $\equiv_{D}$; the set of identity rules, logical rules and structural rules given in Table 9. (Recall that $p$ appearing in $(I d)$ is an atomic propositional formula.) The rules $(T R),(\perp L),\left(W_{L}\right)$ and $\left(W_{R}\right)$ have implicit side-conditions that the structure-position condition must be maintained. The rule (Cut) is called the Elimination Rule (ER) in [3]. This system generates a set of derivable consecutions within the set of all consecutions.

The following theorem shows that $\mathbf{L G L}_{\mathrm{DC}}$ is indeed a display calculus.

Theorem 9.1. The equivalence $\equiv_{D}$ is a display equivalence.

This proof is essentially immediate (although formally by induction) using the rules in Table 8 to shuffle structure and structure connectives across the consecution symbol $\vdash$, and noting that the antecedent/consequent polarity of the displayed part is invariant. The non-commutativity and non-associativity make no substantial difference to the proof in [7], given our choice of display postulates.

Theorem 9.2 (Identity Theorem). The consecution $\phi \vdash \phi$ is derivable for all propositions $\phi$.

The proof is by induction on the structure of $\phi$, and follows that in [7]. Again, the set-up of the rules means that non-commutativity (and non-associativity) of the multiplicatives makes only a trivial change to the proof.

Corollary 9.3. The consecutions $\neg \phi \vdash \phi \rightarrow \perp$ and $\phi \rightarrow \perp \vdash \neg \phi$ and $\neg \neg \phi \vdash \phi$ are derivable.

Thus the connective $\neg$ is a classical negation. The proof of each is a short derivation using the Identity Theorem.

Theorem 9.4. The Cut rule of $\mathbf{L G L}_{\mathrm{DC}}$ is admissible.

Proof. It suffices to verify that Belnap's conditions (C1-C8) hold [3]. This is done in detail in the commutative case in [8]. Conditions $(\mathrm{C} 1-\mathrm{C} 7)$ are treated identically for $\mathbf{L} \mathbf{G L}_{\mathrm{DC}}$, and we omit detailed discussion of them. This leaves the final condition:

- (C8) Eliminability of matching principal formulae. If there are inferences $I_{1}$ and $I_{2}$ with conclusions $\Gamma \vdash \phi$ and $\phi \vdash \Delta$ respectively, and $\phi$ is principal in both inferences, then $\Gamma \vdash \Delta$ is one of $\Gamma \vdash \phi$ or $\phi \vdash \Delta$, or there is a derivation of $\Gamma \vdash \Delta$ using $I_{1}$ and $I_{2}$ in which every instance of $C$ ut has a cut-formula which is a proper subformula of $\phi$.

Verification: If $\phi$ is atomic then $\Gamma=\Delta=\phi$, and the result holds trivially. The other cases are all quite standard (following [8] again) and are those in which the cut-formula $\phi$ is introduced by the final rules of $I_{1}$ and $I_{2}$. 


\section{Identity Rules}

$$
\begin{aligned}
(I d) & \overline{\mathrm{p} \vdash \mathrm{p}} \\
(C u t) & \frac{\Gamma \vdash \phi \quad \phi \vdash \Delta}{\Gamma \vdash \Delta} \\
\left(\equiv_{D}\right) & \frac{\Gamma^{\prime} \vdash \Delta^{\prime}}{\Gamma \vdash \Delta}\left(\Gamma \vdash \Delta \equiv_{D} \quad \Gamma^{\prime} \vdash \Delta^{\prime}\right)
\end{aligned}
$$

\section{Logical Rules}

$$
\begin{aligned}
& (\top L) \frac{\emptyset \vdash \Gamma}{\top \vdash \Gamma} \\
& \overline{\Gamma \vdash \top} \quad(\top R) \\
& (\perp L) \quad \overline{\perp \vdash \Gamma} \\
& \frac{\Gamma \vdash \emptyset}{\Gamma \vdash \perp} \quad(\perp R) \\
& (\wedge L) \frac{\phi ; \psi \vdash \Gamma}{\phi \wedge \psi \vdash \Gamma} \\
& \frac{\Gamma \vdash \phi \quad \Gamma \vdash \psi}{\Gamma \vdash \phi \wedge \psi}(\wedge R) \\
& (\vee L) \frac{\phi \vdash \Gamma \quad \psi \vdash \Gamma}{\phi \vee \psi \vdash \Gamma} \\
& \frac{\Gamma \vdash \phi ; \psi}{\Gamma \vdash \phi \vee \psi} \quad(\vee R) \\
& (\rightarrow L) \frac{\Gamma \vdash \phi \quad \psi \vdash \Delta}{\phi \rightarrow \psi \vdash \# \Gamma ; \Delta} \\
& \frac{\Gamma ; \phi \vdash \psi}{\Gamma \vdash \phi \rightarrow \psi} \quad(\rightarrow R) \\
& (\neg L) \quad \frac{\# \phi \vdash \Gamma}{\neg \phi \vdash \Gamma} \\
& \frac{\Gamma \vdash \# \phi}{\Gamma \vdash \neg \phi} \quad(\neg R) \\
& (-L) \frac{\phi, \psi \vdash \Gamma}{\phi \triangleright \psi \vdash \Gamma} \\
& \frac{\Gamma \vdash \phi \quad \Delta \vdash \psi}{\Gamma, \Delta \vdash \phi \triangleright \psi} \quad(\triangleright R) \\
& (\rightarrow L) \frac{\Gamma \vdash \phi \quad \psi \vdash \Delta}{\phi \rightarrow \psi \vdash \Gamma \multimap \Delta} \\
& \frac{\Gamma, \phi \vdash \psi}{\Gamma \vdash \phi \rightarrow \psi} \quad(\rightarrow R) \\
& \left(\llcorner L) \frac{\Gamma \vdash \phi \quad \psi \vdash \Delta}{\phi \vdash \psi \vdash \Gamma \bullet \Delta}\right. \\
& \frac{\phi, \Gamma \vdash \psi}{\Gamma \vdash \phi} \psi(-R)
\end{aligned}
$$

\section{Structural Rules}

$$
\begin{array}{llr}
\left(W_{L}\right) & \frac{\Gamma \vdash \Delta}{\Gamma ; \Gamma^{\prime} \vdash \Delta} & \frac{\Gamma \vdash \Delta}{\Gamma \vdash \Delta ; \Delta^{\prime}}\left(W_{R}\right) \\
\left(C_{L}\right) & \frac{\Gamma ; \Gamma \vdash \Delta}{\Gamma \vdash \Delta} & \frac{\Gamma \vdash \Delta ; \Delta}{\Gamma \vdash \Delta}\left(C_{R}\right) \\
\left(A_{L}\right) & \frac{\Gamma ;(\Theta ; \Lambda) \vdash \Delta}{\overline{(\Gamma ; \Theta) ; \Lambda \vdash \Delta}} & \frac{\Gamma \vdash \Delta ;(\Theta ; \Lambda)}{\Gamma \vdash(\Delta ; \Theta) ; \Lambda}\left(A_{R}\right) \\
(\emptyset L) & \frac{\bar{\Gamma} ; \Gamma \vdash \Delta}{\Gamma \vdash \Delta} & \frac{\Gamma \vdash \Delta ; \emptyset}{\Gamma \vdash \Delta}(\emptyset R)
\end{array}
$$

TABLE 9. Rules of $\mathbf{L} \mathbf{G} \mathbf{L}_{\mathrm{DC}}$ 
By way of illustration, we give the case of the right multiplicative implication. A derivation of the form

$$
\frac{\frac{\Gamma, \phi \vdash \psi}{\Gamma \vdash \phi \rightarrow \psi}(\rightarrow R) \quad \frac{\Delta \vdash \phi \quad \psi \vdash \Theta}{\phi \rightarrow \psi \vdash \Delta \rightarrow \Theta}}{\Gamma \vdash \Delta \rightarrow \Theta}(C u t)
$$

can be replaced by a derivation

$$
\begin{gathered}
\frac{\Gamma, \phi \vdash \psi \quad \psi \vdash \Theta}{\Gamma, \phi \vdash \Theta}(C u t) \\
\frac{\Delta \vdash \phi \quad \frac{\phi \vdash \Gamma \bullet-\Theta}{\phi \vdash}\left(\equiv_{D}\right)}{\frac{\Delta \vdash \Gamma \bullet \Theta}{\Gamma, \Delta \vdash \Theta}\left(\equiv_{D}\right)}\left(\equiv_{D}\right) \\
\frac{\Gamma \vdash \Delta \bullet \Theta}{\Gamma-\Delta}\left(\equiv_{D}\right)
\end{gathered}
$$

that uses only cuts on proper sub-formulae of the original cut-formula $\phi \rightarrow \psi$. Note that it is the left structural implication connective $\bullet-$ that is used to do the temporary symbol shuffling in this case. This display calculus appears to need both multiplicative implications, not just one or the other.

We now relate the four different logical calculi.

Theorem 9.5. The following four statements hold:

A. Each axiom of $\mathbf{L} \mathbf{G} \mathbf{L}_{\mathrm{H}}$ is derivable in $\mathbf{L} \mathbf{G} \mathbf{L}_{\mathrm{ND}}$;

B. Each rule of $\mathbf{L} \mathbf{G} \mathbf{L}_{\mathrm{H}}$ is derivable in $\mathbf{L} \mathbf{G} \mathbf{L}_{\mathrm{ND}}$;

C. Each rule of $\mathbf{L} \mathbf{G} \mathbf{L}_{\mathrm{ND}}$ is derivable in $\mathbf{L} \mathbf{G} \mathbf{L}_{\mathrm{SC}}$;

D. Each rule of $\mathbf{L} \mathbf{G} \mathbf{L}_{\mathrm{SC}}$ is derivable in $\mathbf{L} \mathbf{G} \mathbf{L}_{\mathrm{DC}}$.

Proof. We consider the four statements A-D in turn.

Statements A and B. These facts are well-known for Boolean BI, and the proofs here are almost identical.

Statement C. Again, the proof here is essentially standard; the non-commutativity and nonassociativity of multiplicatives make no real difference as the relation $\equiv$ and the rule (E) are weakened in the same way for both calculi, and (following careful design) the other rules do not make use of these properties. For an example of one rule, consider the $(\rightarrow E)$ rule of $\mathbf{L} \mathbf{G L}_{\mathrm{ND}}$. The following proof-figure in $\mathbf{L} \mathbf{G} \mathbf{L}_{\mathrm{SC}}$ derives the conclusion of $(\rightarrow E)$ from its premisses:

$$
\frac{\Gamma \vdash \phi \rightarrow \psi \quad \frac{\Delta \vdash \phi \quad \overline{\psi \vdash \psi}}{\phi \rightarrow \psi, \Delta \vdash \psi}}{\Gamma, \Delta \vdash \psi}\left(\begin{array}{l}
(\mathrm{Id}) \\
(\mathrm{Cut}) .
\end{array}\right.
$$

Statement D. First, observe that all bunches are structures, so that bunches appearing in $\mathbf{L} \mathbf{G} \mathbf{L}_{\mathrm{SC}}$ rules are meaningful in $\mathbf{L} \mathbf{G} \mathbf{L}_{\mathrm{DC}}$ rules. We then consider each rule in turn. Some of these cases require repeated use of $\left(\equiv_{D}\right)$ because the display calculus has the rule stated in a 'shallow' way, where the sequent calculus has it stated in a 'deep' way. In each such case the evident associated induction is omitted. The cases are as follows:

- The $(I d)$ rule of $\mathbf{L G L}_{\mathrm{SC}}$ is derivable by the Identity Theorem;

- $(R A A)$ is derivable by Corollary 9.3 and $(C u t)$ of $\mathbf{L} \mathbf{G} \mathbf{L}_{\mathrm{DC}}$;

- $(\wedge R)$ of $\mathbf{L} \mathbf{G L}_{\mathrm{SC}}$ is derivable using $\left(W_{L}\right)$ and $(\wedge R)$ of $\mathbf{L} \mathbf{G} \mathbf{L}_{\mathrm{DC}}$;

- $(\vee R)$ of $\mathbf{L} \mathbf{G} \mathbf{L}_{\mathrm{SC}}$ is derivable using $\left(W_{R}\right)$ and $(\vee R)$ of $\mathbf{L} \mathbf{G} \mathbf{L}_{\mathrm{DC}}$;

- $(\top R),(\rightarrow R),(\rightarrow R),\left(\llcorner R)\right.$ and $(\neg R)$ of $\mathbf{L} \mathbf{G L}_{\mathrm{SC}}$; are each derivable using the corresponding rule of $\mathbf{L} \mathbf{G L}_{\mathrm{DC}}$; 


$$
\begin{array}{lll}
\Phi_{\phi}=\phi=\Psi_{\phi} & \\
\Phi_{\emptyset}=\top & \Phi_{\# \Gamma}=\neg \Psi_{\Gamma} & \Phi_{\Gamma ; \Delta}=\Phi_{\Gamma} \wedge \Phi_{\Delta} \\
\Phi_{\Gamma, \Delta}=\Phi_{\Gamma} \triangleright \Phi_{\Delta} & \Phi_{\Gamma \bullet \Delta} \text { undefined } & \Phi_{\Gamma \bullet \Delta} \text { undefined } \\
\Psi_{\emptyset}=\perp & \Psi_{\# \Gamma}=\neg \Phi_{\Gamma} & \Psi_{\Gamma ; \Delta}=\Psi_{\Gamma} \vee \Psi_{\Delta} \\
\Psi_{\Gamma, \Delta} \text { undefined } & \Psi_{\Gamma \bullet \Delta}=\Phi_{\Gamma}\left\llcorner\Psi_{\Delta}\right. & \Psi_{\Gamma \bullet \Delta}=\Phi_{\Gamma} \rightarrow \Psi_{\Delta}
\end{array}
$$

TABLE 10. Antecedent and Consequent Formulae

- $(\perp L),(\top L), \wedge L,(\vee L),(\triangleright L),(C u t)(\rightarrow L),(\rightarrow L),\left(\llcorner L)\right.$ of $\mathbf{L} \mathbf{G} \mathbf{L}_{\mathrm{SC}}$ are derivable using $\left(\equiv_{D}\right)$ repeatedly and the corresponding rule of $\mathbf{L} \mathbf{G} \mathbf{L}_{\mathrm{DC}}$;

- $(C)$ is derivable using $\left(\equiv_{D}\right)$ repeatedly and $\left(C_{L}\right)$;

- $(W)$ is derivable using $\left(\equiv_{D}\right)$ repeatedly and $\left(W_{L}\right)$;

- $(E)$ is derivable, since if $\Gamma$ and $\Delta$ are equivalent bunches, $\Gamma \equiv \Delta$, and $\Gamma \vdash \phi$ in $\mathbf{L G} \mathbf{G}_{\mathrm{SC}}$, then a finite number of applications of $\left(\equiv_{D}\right),\left(A_{L}\right)$ and $(\emptyset L)$ can be used to derive $\Delta \vdash \phi$.

Let $\Gamma$ be any structure. Define, where possible, the antecedent formula, $\Phi_{\Gamma}$, and consequent formula, $\Psi_{\Gamma}$, by simultaneous recursion on the structure of $\Gamma$ as in Table 10.

Theorem 9.6. Let $\frac{\left(\Gamma_{j} \vdash \Delta_{j}\right)_{j \in J}}{\Gamma^{\prime} \vdash \Delta^{\prime}}$ be a rule of $\mathbf{L} \mathbf{G L}_{\mathrm{DC}}$, where $J$ is a finite index set. If $J$ is empty, then for any instance of $\Gamma^{\prime} \vdash \Delta^{\prime}$ we have that $\Phi_{\Gamma^{\prime}} \vdash \Psi_{\Delta^{\prime}}$ is an instance of an axiom of $\mathbf{L} \mathbf{G} \mathbf{L}_{\mathrm{H}}$. If $J$ is non-empty and $\Phi_{\Gamma_{j}}$ and $\Psi_{\Delta_{j}}$ are defined for all $j \in J$, then $\frac{\left(\Phi_{\Gamma_{j}} \vdash \Psi_{\Delta_{j}}\right)_{j \in J}}{\Phi_{\Gamma^{\prime}} \vdash \Psi_{\Delta^{\prime}}}$ is derivable in LGL $\mathbf{L}_{\mathrm{H}}$.

Proof. We consider cases of rules of $\mathbf{L G L}_{\mathrm{DC}}$.

- $(I d),(\top R),(\perp L)$ are instances of the case where $J$ is empty, and follow from 1, 2, 3 of $\mathbf{L G L}_{\mathrm{H}}$, respectively.

- $(\top L),(\perp R),(\wedge L),(\vee R),(\neg L),(\neg R),(\neg L),(\neg R)$ are translated so that the conclusion is the same as the premiss, and the rule $\frac{\phi \vdash \phi}{\phi \vdash \phi}$ is derivable in $\mathbf{L} \mathbf{G L}_{\mathrm{H}}$.

- $(\wedge R),(\vee L),(\rightarrow R),(\rightarrow R),\left(\llcorner R),\left(W_{L}\right),\left(W_{R}\right)\right.$ translate directly to (instances of) Rules $5,7,9,13,15,11,8$ of $\mathbf{L G L}_{\mathrm{H}}$, respectively.

- $(\vee R),(\rightarrow L),\left(C_{L}\right),\left(C_{R}\right),\left(A_{L}\right),\left(A_{R}\right),(\emptyset L)$ and $(\emptyset R)$ are standard derivations in Hilbert calculi for bunched systems, not requiring the use of rules dealing with multiplicative connectives, and are omitted.

- $(C u t)$ translates directly.

- $(\rightarrow L)$ follows because the derivation below is possible in $\mathbf{L} \mathbf{G L}_{\mathrm{H}}$

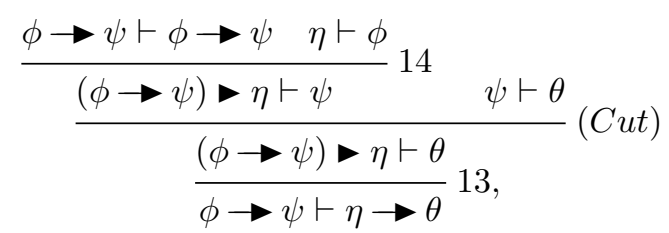

for any $\phi, \psi, \eta, \theta$, and the $(\llcorner L)$ case is similar.

- $\left(\equiv_{D}\right)$ is generated from the display identities; each of the display identities is easily verified to translate into a derivable rule of $\mathbf{L} \mathbf{G L}_{\mathrm{H}}$; therefore, by induction on the minimum number of identities required to establish the particular instance of $\equiv_{D}$ used in the $\left(\equiv_{D}\right)$ instance, the result holds in this case. 


\section{Discussion}

In this paper we considered, informally, just a simple notion of action in order to explore our examples, one can readily propose to formulate a Hennessy-Milner-style version of LGL, just as the bunched modal logic MBI [13, 11, 12] is a Hennessy-Milner-style version of the bunched logic BI [35]. The logic MBI employs a judgement $L, R, E \vDash \phi$, which can be understood as 'property $\phi$ holds of process $E$ executing relative to resources $R$ at locations $L$ '. In the proposed logic, $L$ would be a graph of the kind discussed in this paper, having vertices labelled with resources.

As well as providing a setting for a thorough analysis of examples much richer than the two considered above, such a framework might provide a basis for exploring logical characterizations of the structural properties of layered graphs (i.e., a modal 'correspondence theory' of layering). Examples of properties might include the layering structure itself and distribution properties of resources around the location graphs. An alternative development would be to extend LGL in the style of dynamic logic [25, 26].

The examples presented make use of resources moving around locations. It would also be interesting to explore situations where the underlying graph architecture is altered. There are again many ready examples of such situations in security.

\section{REFERENCES}

[1] A.R. Anderson and N.D. Belnap. Entailment: the Logic of Relevance and Necessity, volume I. Princeton University Press, 1975.

[2] A.R. Anderson, J.M. Dunn, and N.D. Belnap. Entailment: the Logic of Relevance and Necessity, volume II. Princeton University Press, 1992.

[3] N.D. Belnap. Display logic. Journal of Philosophical Logic, 11:375-414, 1982.

[4] G.M. Bierman. What is a categorical model of intuitionistic linear logic? In Proceedings of Second International Conference on Typed $\lambda$-calculi and Applications, volume 902 of Lecture Notes in Computer Science, pages 78 93. Springer-Verlag, Berlin, 1995.

[5] Ken Binmore. Playing for Real. Oxford University Press, 2007.

[6] P. Bródka, K. Skibicki, P. Kazienko, and K. Musiał. A degree centrality in multi-layered social network. In Int. Conference on Computational Aspects of Social Networks, 2011.

[7] James Brotherston. Bunched logics displayed. Studia Logica: Special Issue on Recent Developments related to Residuated Lattices and Substructural Logics, 100(6):1223-1254, 2012.

[8] James Brotherston and Cristiano Calcagno. Classical BI: Its semantics and proof theory. Logical methods in Computer Science, 6(3:3):1-42, 2010.

[9] L. Cardelli, P. Gardner, and G. Ghelli. A spatial logic for querying graphs. In ICALP: Automata, Languages, and Programming, 29th International Colloquium, volume 2380 of LNCS, pages 597-610, 2002.

[10] Luca Cardelli and Andrew D. Gordon. Anytime, anywhere. modal logics for mobile ambients. In Proceedings of the 27th ACM Symposium on Principles of Programming Languages, pages 365-377, 2000.

[11] M. Collinson, B. Monahan, and D. Pym. A logical and computational theory of located resource. Journal of Logic and Computation, 19(b):1207-1244, 2009.

[12] M. Collinson, B. Monahan, and D. Pym. A Discipline of Mathematical Systems Modelling. College Publications, 2012.

[13] M. Collinson and D. Pym. Algebra and logic for resource-based systems modelling. Mathematical Structures in Computer Science, 19:959-1027, 2009. doi:10.1017/S0960129509990077.

[14] Matthew Collinson, Brian Monahan, and David Pym. Semantics for structured systems modelling and simulation. In Proc. Simutools 2010. ACM Digital Library, ISBN 78-963-9799-87-5, 2010.

[15] Bruno Courcelle. The expression of graph properties and graph transformations in monadic second-order logic. Graph grammars and computing by graph transformations, 1:313-400, 1997.

[16] A. Dawar, P. Gardner, and G. Ghelli. Expressiveness and complexity of graph logic. Information and Computation, 205:236-310, 2007.

[17] K. Došen. Sequent systems and groupoid models I. Studia Logica, 47:353-385, 1988.

[18] K. Došen. Sequent systems and groupoid models II. Studia Logica, 48:41-65, 1989.

[19] A. Fiat, D. Foster, H. Karloff, Y. Rabani, Y. Ravid, and S. Vishwanathan. Competitive algorithms for layered graph traversal. SIAM Journal on Computing, 28(2):447-462, 1998.

[20] D. Galmiche, D. Méry, and D. Pym. The Semantics of BI and Resource Tableaux. Mathematical Structures in Computer Science, 15:1033-1088, 2005.

[21] G. Gentzen. Untersuchungen über das logische Schliessen. Mathematische Zeitschrift, 39:176-210, 405-431, 1934 .

[22] J.-Y. Girard. Linear logic. Theoretical Computer Science, 50(1):1-102, 1987. 
[23] L. Gouveia, A. Paias, and D. Sharma. Modeling and solving the rooted distance-constrained minimum spanning tree problem. Computers and Operations Res., 35(2):600-613, 2008.

[24] L. Gouveia, L. Simonetti, and E. Uchoa. Modeling hop-constrained and diameter-constrained minimum spanning tree problems as steiner tree problems over layered graphs. Mathematical Programming, pages 1-26, 2010.

[25] D. Harel. Dynamic logic. In D. Gabbay and F. Guenthner, editors, Handbook of Philosophical Logic, Volume II, pages 497-604. Dordrecht: D. Reidel, 1984.

[26] David Harel, Dexter Kozen, and Jerzy Tiuryn. Dynamic Logic. MIT Press, 2000.

[27] M. Hennessy and R. Milner. Algebraic laws for nondeterminism and concurrency. Journal of the ACM, 32(1):137-161, 1985.

[28] S.S. Ishtiaq and P. O'Hearn. BI as an assertion language for mutable data structures. In 28th ACM-SIGPLAN Symposium on Principles of Programming Languages, London, pages 14-26. Association for Computing Machinery, 2001.

[29] M. Kurant and P. Thiran. Layered complex networks. Physical Review Letters, 96:138701(4), 2006.

[30] J. Lambek. Deductive Systems and Categories I. J. Math. Systems Theory, 2:278-318, 1968.

[31] J. Lambek. Deductive Systems and Categories II. Springer LNM, 86:76-122, 1969.

[32] J. Lambek. Deductive Systems and Categories III. Springer LNM, 274:57-82, 1972

[33] J. Lambek. From categorical grammar to bilinear logic. In P. Schroeder-Heister and K. Došen, editors, Substructural Logics, pages 207-237. Oxford University Press, 1993.

[34] R. Milner. Communication and Concurrency. Prentice Hall, New York, 1989.

[35] P.W. O'Hearn and D.J. Pym. The logic of bunched implications. Bulletin of Symbolic Logic, 5(2):215-244, June 1999.

[36] C. Papadimitriou and M. Yannakakis. Shortest paths without a map. Theoretical Computing Science, 84(1):127-150, 1991.

[37] A. Paz. A theory of decomposition into prime factors of layered interconnection networks. Discrete Applied Mathematics, 159(7):628-646, 2011.

[38] D. Prawitz. Natural Deduction: A Proof-Theoretical Study. Almquist and Wiksell, Stockholm, 1965.

[39] David Pym. On Bunched Predicate Logic. In Proc. 14th Symposium on Logic in Computer Science, pages 183-192. IEEE Computer Society Press, 1999.

[40] D.J. Pym. The Semantics and Proof Theory of the Logic of Bunched Implications, volume 26 of Applied Logic Series. Kluwer Academic Publishers, 2002. Errata and Remarks maintained at publisher's website and at: http://www.cantab.net/users/david.pym/BI-monograph-errata.pdf.

[41] D.J. Pym, P.W. O'Hearn, and H. Yang. Possible Worlds and Resources: The Semantics of BI. Theoretical Computer Science, 315(1):257-305, 2004.

[42] S. Read. Relevant Logic: A Philosophical Examination of Inference. Basil Blackwell, 1988.

[43] G. Restall. An Introduction to Substructural Logics. Routledge, 1999.

[44] John Reynolds. Separation logic: A logic for shared mutable data structures. In Proceedings of the Seventeenth Annual IEEE Symposium on Logic in Computer Science, Copenhagen, Denmark, July 22-25, 2002, pages 55-74. IEEE Computer Society Press, 2002.

[45] B. Schneier. Schneier on Security: A blog covering security and security technology, February 2005. http: //www.schneier.com/blog/archives/2005/02/the_weakest_lin.html.

[46] J. Wang, P. De Wilde, and H. Wang. Topological analysis of a two coupled evolving networks model for business systems. Expert Systems with Applicns., 36:9548-9556, 2009.

Matthew Collinson, University of Aberdeen

E-mail address: matthew.collinson@abdn.ac.uk

Kevin McDonald, University of Aberdeen

E-mail address: kevin.mcdonald@abdn.ac.uk

DAVID PYM, UCL

E-mail address: d.pym@ucl.ac.uk 